\title{
The Green function for elliptic systems in two dimensions
}

\author{
J.L. Taylor \\ Department of Mathematics \\ Murray State University \\ Murray, Kentucky 42071, USA \\ S. Kim * \\ Department of Computational Science and Engineering \\ Yonsei University \\ Seoul, Korea \\ R.M. Brown ${ }^{\dagger}$ \\ Department of Mathematics \\ University of Kentucky \\ Lexington, Kentucky 40506, USA
}

\begin{abstract}
We construct the fundamental solution or Green function for a divergence form elliptic system in two dimensions with bounded and measurable coefficients. We consider the elliptic system in a Lipschitz domain with mixed boundary conditions. Thus we specify Dirichlet data on part of the boundary and Neumann data on the remainder of the boundary. We require a corkscrew or non-tangential accessibility condition on the set where we specify Dirichlet boundary conditions. Our proof proceeds by defining a variant of the space $B M O(\Omega)$ that is adapted to the boundary conditions and showing that the solution exists in this space. We also give a construction of the Green function with Neumann boundary conditions and the fundamental solution in the plane.
\end{abstract}

\footnotetext{
* Seick Kim is supported by NRF Grant No. 2010-0008224 and R31-10049 (WCU program).

${ }^{\dagger}$ This work was partially supported by a grant from the Simons Foundation (\#195075 to Russell Brown).
} 


\section{Introduction}

We consider a weak formulation of the mixed problem for a second-order elliptic operator in a bounded, connected, and open set $\Omega$ in $\mathbf{R}^{2}$. To state the mixed problem, we fix a decomposition of the boundary $\partial \Omega=D \cup N$ with $D \cap N=$ $\emptyset$. We let $L$ be an elliptic operator in divergence form with bounded and measurable coefficients and we consider the boundary value problem

$$
\begin{cases}L u=f & \text { in } \Omega \\ u=0 & \text { on } D \\ \frac{\partial u}{\partial \nu}=f_{N}, & \text { on } N .\end{cases}
$$

where $\partial / \partial \nu$ is the natural co-normal derivative associated with the operator $L$. We will require that $\Omega$ be a Lipschitz domain and that the set $D$ satisfy a corkscrew condition (or non-tangential accessibility condition) as in [25]. We will give a precise formulation of the mixed problem in section 2. We emphasize that our results apply to elliptic systems where $L$ acts on vector-valued functions as well as equations. Our goal is to give a construction of the matrix-valued Green function for this boundary condition and show that the Green function satisfies the estimates

$$
\begin{aligned}
|G(x, y)| & \leq C(1+\log (d /|x-y|)), \quad x, y \in \Omega \\
|G(x, y)-G(x, z)| & \leq C \frac{|y-z|^{\gamma}}{|x-y|^{\gamma}}, \quad x, y, z \in \Omega,|y-z|<\frac{1}{2}|x-y| \\
|G(x, y)| & \leq \frac{\operatorname{dist}(y, D)^{\gamma}}{|x-y|^{\gamma}}, \quad \operatorname{dist}(y, D)<\frac{1}{2}|x-y| .
\end{aligned}
$$

where $d$ is the diameter of $\Omega$ and the constant $C$ and $\gamma>0$ depend only on assumptions on the operator, the domain, and the decomposition of the boundary.

Given the logarithmic singularity of the Green function, it is natural to look for a Green function in the space of functions of bounded mean oscillation. We fix a set $D$ and define the space $B M O_{D}(\Omega)$ which consists of functions in $B M O(\Omega)$ and which vanish in an appropriate sense as we approach $D$. We will construct the Green function in this space and then obtain the pointwise estimates.

There is a great deal of literature on the existence of Green functions and we do not try to summarize it all here. Littman, Stampacchia, and Weinberger [18] establish that the Green function has a logarithmic singularity in two dimensions. Grüter and Widman [14] give a nice construction of the Green function for the Dirichlet problem in dimensions three and higher and treat operators that are not self-adjoint. Kenig and Pipher [17] give a construction of the Neumann function or the Green function for the Neumann problem when $n \geq 3$. The existence of a global fundamental solution for an elliptic operator in the plane was established by Kenig and Ni [16]. Using their results, Chanillo and $\mathrm{Li}$ 4] show that the global fundamental solution lies in $B M O\left(\mathbf{R}^{2}\right)$. 
The results of the previous paragraph are for single equations. More recently, there has been interest in constructing Green functions for elliptic systems. In this case, we are only able to obtain upper bounds. The work of Auscher and collaborators [1] establishes the existence of a Green function for operators with complex coefficients (which may be viewed as a system of equations with real coefficients). This work was motivated in part by their interest in the Kato problem. Dolzmann and Müller [7] construct the Green function in a $C^{1}$-domain for an elliptic operator with continuous coefficients. Dong and Kim [8] establish the existence of a Green function for elliptic systems which are not assumed to be self-adjoint under the hypothesis that solutions of the operator are bounded or Hölder continuous. Their argument gives Gaussian upper bounds for the parabolic Green function and then integrates the parabolic Green function in time giving the Green function for the elliptic problem. Recently, Choi and Kim [5] have given a construction of the Neumann function in dimensions three and higher under the assumption that solutions of the operator satisfy local Hölder continuity and boundedness estimates. In two dimensions, the necessary results on the Hölder continuity of solutions dates back to Morrey [21]. A paper of Calanchi, Rodino, and Tri [2] observes that the Green function for the Dirichlet problem for the Laplacian lies in $B M O$ for planar domains. Their proof relies on the maximum principle which is not available to treat the systems considered here. In addition to our main goal of treating the Green function for the mixed problem, the present paper complements the work of Choi and Kim by providing a construction of the Green function for the Neumann problem in two dimensions. Recent work of D. Mitrea and I. Mitrea [20] gives the existence of the Dirichlet Green function for various constant coefficient operators in Lipschitz domains.

The present paper was motivated by an effort to construct the Green function for elliptic systems under mixed boundary conditions. Taylor, Ott, and Brown [25] give a construction of the Green function for a class of mixed problems for the Laplacian in a Lipschitz domain in dimensions two and higher. Their argument begins with the free space fundamental solution and uses a reflection to first construct a Green function for Neumann boundary conditions and finally corrects the boundary conditions to give a Green function for mixed boundary conditions. Below, we provide a new proof of their results in two dimensions and establish the existence of a Green function with mixed boundary conditions for a large class of elliptic systems in two dimensions. We expect that the Green function constructed below will be useful in extending Taylor, Ott, and Brown's results on the $L^{p}$-mixed problem for the Laplacian to systems in two dimensions. It is an interesting, open question to study the mixed problem for elliptic systems in dimensions three and higher. The main difficulty in carrying out this extension is the lack of estimates for the Green function.

The construction of the Green function for an operator $L$ is closely related to local, scale-invariant estimates for solutions of $L$. In the case of a single equation, the Hölder continuity of solutions to the mixed problem was established by Stampacchia [24] using the method of De Giorgi. Stampacchia considers 
the mixed problem in domains which locally are equivalent by a bi-Lipschitz transformation to a mixed problem in a half-space with the boundary between $D$ and $N$ a hyperplane. A more recent work of Gröger [13] uses the method of Meyers [19] to show that solutions of the mixed problem with nice data satisfy $\nabla u \in L^{2+\epsilon}(\Omega)$. Gröger's assumptions on the domain and the decomposition of the boundary are similar to those of Stampacchia. When considering the more general decompositions of the boundary introduced by Taylor, Ott, and Brown and considered in this paper, it seems to be simpler to use the method of reverse Hölder inequalities as in Gehring [9] and Giaquinta and Modica [1] to obtain that $\nabla u \in L^{2+\epsilon}(\Omega)$.

Our formulation of the mixed problem allows for the extreme cases where $D=\emptyset$ or $D=\partial \Omega$ which give the Neumann problem and the Dirichlet problem, respectively. The Green function for the Dirichlet problem is treated alongside the mixed problem in section 4. The properties of the Green function are given in Theorem 4.1. The changes needed for the Neumann problem appear in section [5] and the properties of the Green function for the Neumann problem are given in Theorem 5.14. In Section 6, we provide a proof of the existence of a fundamental solution in the plane. The construction of a global fundamental solution for an equation was perhaps known, however the detailed construction appears to have first been written down in a paper of Kenig and Ni from 1985 [16]. We provide a different construction of the fundamental solution which also applies to systems. The existence of a fundamental solution in the plane and the properties of this fundamental solution are given in Theorem 6.7. The Lamé system (with variable Lamé parameters) provides a family of examples to illustrate the use of our results. We describe this system in section 2 and show that it satisfies the hypotheses of our main results.

\section{Preliminary material and the weak for- mulation of the mixed problem}

We will consider the Green function for boundary value problems in a bounded Lipschitz domain $\Omega$ and we begin by giving the definition of these domains. Given a constant $M>0, x \in \partial \Omega$, and $r>0$, we let $Z_{r}(x)=\left\{y:\left|y_{1}-x_{1}\right|<\right.$ $\left.r,\left|y_{2}-x_{2}\right|<(3 M+1) r\right\}$. We say that $Z_{r}(x)$ is a coordinate rectangle for $\Omega$ if there is a Lipschitz function $\phi: \mathbf{R} \rightarrow \mathbf{R}$ so that

$$
\begin{aligned}
\Omega \cap Z_{r}(x) & =\left\{y: y_{2}>\phi\left(y_{1}\right)\right\} \cap Z_{r}(x) \\
\partial \Omega \cap Z_{r}(x) & =\left\{y: y_{2}=\phi\left(y_{1}\right)\right\} \cap Z_{r}(x)
\end{aligned}
$$

We assume that the coordinate system used in this coordinate rectangle is a rotation of the standard coordinate system. We say that $\Omega$ is a Lipschitz domain if $\Omega$ is a bounded connected open set and for each $x \in \partial \Omega$, we may find a coordinate rectangle centered at $x$. Since the boundary is compact, we may cover $\partial \Omega$ by a finite collection of coordinate rectangles $\left\{Z_{r_{i}}\left(x_{i}\right)\right\}_{i=1}^{N}$ so that each 
$Z_{100 r_{i}}\left(x_{i}\right)$ is also a coordinate rectangle. We set $r_{0}=\min \left\{r_{i}: i=1, \ldots, N\right\}$ and we will use this value as a characteristic length of the domain when we state scale-invariant estimates.

For $x \in \bar{\Omega}$ and $\rho \in\left(0,4 r_{0}\right)$, we define local domains $\Omega_{\rho}(x)$ which will play the role of disks in our work. In the case that $\operatorname{dist}(x, \partial \Omega)>\rho$, we let $\Omega_{\rho}(x)=B_{\rho}(x)$, the disk centered at $x$ with radius $\rho$. In the case that $\operatorname{dist}(x, \partial \Omega) \leq \rho$, we pick a coordinate rectangle containing $x=\left(x_{1}, x_{2}\right)$ and using the coordinates for this rectangle, we let $\hat{x}=\left(x_{1}, \phi\left(x_{1}\right)\right)$ be obtained by projecting onto the boundary and define $\Omega_{\rho}(x)=Z_{\rho}(\hat{x}) \cap \Omega$.

For $x \in \partial \Omega$ and $\rho \in\left(0, r_{0}\right)$, we define a boundary interval $\Delta_{\rho}(x)$ by $\Delta_{\rho}(x)=Z_{\rho}(x) \cap \partial \Omega$. The domains $\Omega_{\rho}(x)$ are star-shaped Lipschitz domains and the boundary intervals $\Delta_{\rho}(x)$ are connected. These properties are helpful in establishing the Poincaré and Korn inequalities introduced below. For this reason, we prefer them to $B_{\rho}(x) \cap \Omega$ and $B_{\rho}(x) \cap \partial \Omega$ used in [23, 25], for example. There is a price to pay as given $x$ and $\rho$ we will have several choices for $\Delta_{\rho}(x)$ and $\Omega_{\rho}(x)$. Our results will hold for any such choice provided that when several of these objects occur in an estimate we use the same coordinate rectangle to define each of them.

We let $D \subset \partial \Omega$ be the set where we specify the Dirichlet data and then we put $N=\partial \Omega \backslash D$. We will require that $D$ satisfy the interior corkscrew condition. This means that for each $x \in \partial D$ (where the boundary is taken with respect to $\partial \Omega)$ and $r \in\left(0, r_{0}\right)$, we may find $x_{r} \in D$ so that

$$
\left|x-x_{r}\right| \leq r \text { and } \operatorname{dist}\left(x_{r}, N\right) \geq M^{-1} r .
$$

As our boundary is locally a Lipschitz graph, this easily implies that for each $x \in D$ and $r \in\left(0, r_{0}\right)$, we have that $\sigma\left(\Delta_{r}(x) \cap D\right) \geq c r$ (see [25, Section 1] for details). Note that if $D$ is not empty, then the corkscrew condition implies that the interior of $D$ is not empty.

Now we turn to the precise description of the boundary value problem. As we are considering an operator acting on vector-valued functions, most of the functions we consider will take values in $\mathbf{R}^{m}$ for some $m$. We do not explicitly denote the target in our notation for function spaces. However, we emphasize that in the definitions below, all of the functions will be vectorvalued. We let $\dot{W}^{1, p}(\Omega)$ denote the homogeneous Sobolev space of functions having one derivative in $L^{p}(\Omega)$ with the norm $\|u\|_{\dot{W}_{D}^{1, p}(\Omega)}=\|\nabla u\|_{L^{p}(\Omega)}$. Given a set $D \subset \partial \Omega$, we let $\dot{W}_{D}^{1, p}(\Omega)$ denote the subspace obtained by taking the closure in $\dot{W}^{1, p}(\Omega)$ of the smooth functions in $\bar{\Omega}$ which vanish in a neighborhood of $D$. Thus, the elements in $\dot{W}_{D}^{1, p}(\Omega)$ are functions which in some sense vanish on $D$. When $D$ is not empty and satisfies the corkscrew condition and $1<p<\infty$, we have that if $u \in \dot{W}_{D}^{1, p}(\Omega)$, then

$$
r_{0}^{-1 / p}\left(\int_{\partial \Omega}|u|^{p} d \sigma\right)^{1 / p}+r_{0}^{-2 / p}\left(\int_{\Omega}|u|^{p} d y\right)^{1 / p} \leq C\left(\int_{\Omega}|\nabla u|^{2} d y\right)^{1 / 2} .
$$

for a constant $C$ which depends on $M, p$, and $\Omega$. We will let $\dot{W}_{D}^{-1, t^{\prime}}(\Omega)$ be 
the dual of $\dot{W}_{D}^{1, t}(\Omega)$ when $1 \leq t<\infty$. The space $\dot{W}_{D}^{1 / 2,2}(\partial \Omega)$ is defined to be the image of $\dot{W}_{D}^{1,2}(\Omega)$ under the trace map and then $\dot{W}_{D}^{-1 / 2,2}(\partial \Omega)$ the dual of $\dot{W}_{D}^{1 / 2,2}(\partial \Omega)$. We let $\langle\cdot, \cdot\rangle: \dot{W}_{D}^{-1,2}(\Omega) \times \dot{W}_{D}^{1,2}(\Omega) \rightarrow \mathbf{R}$ and $\langle\cdot, \cdot\rangle_{\partial \Omega}:$ $\dot{W}_{D}^{-1 / 2,2}(\partial \Omega) \times \dot{W}_{D}^{1 / 2,2}(\partial \Omega) \rightarrow \mathbf{R}$ be the pairings of duality.

The operator $L$ will act on vector-valued functions in the plane. Formally, $L$ is given by

$$
(L u)^{\alpha}=\sum_{i, j=1}^{2} \sum_{\beta=1}^{m} \frac{\partial}{\partial x_{i}} a_{\alpha \beta}^{i j} \frac{\partial u^{\beta}}{\partial x_{j}}, \quad \alpha=1, \ldots, m .
$$

The coefficients $a_{\alpha \beta}^{i j}$ are assumed to be real, bounded, and measurable functions

$$
\max \left\{\left\|a_{\alpha \beta}^{i j}\right\|_{L^{\infty}(\Omega)}: i, j=1,2, \alpha, \beta=1, \ldots, m\right\} \leq M .
$$

We do not assume a symmetry condition on the coefficients and thus the operator $L$ will not be self-adjoint. We will define $L u$ as an element of $\dot{W}_{D}^{-1,2}(\Omega)$ via the quadratic form

$$
A(u, \phi)=\int_{\Omega} a_{\alpha \beta}^{i j} \frac{\partial u^{\beta}}{\partial x_{j}} \frac{\partial \phi^{\alpha}}{\partial x_{i}} d x .
$$

Here and throughout this paper, we follow the convention that we sum on repeated indices. Since the coefficients are bounded, for some constant $C=$ $C(M, m)$ we have

$$
|A(u, \phi)| \leq C\|u\|_{\dot{W}^{1,2}(\Omega)}\|\phi\|_{\dot{W}^{1,2}(\Omega)} .
$$

We assume the following local ellipticity condition. If $x \in \bar{\Omega}, \rho \in\left(0, r_{0}\right)$, and $u \in \dot{W}_{\emptyset}^{1,2}(\Omega)$, then for all constant vectors $c \in \mathbf{R}^{m}$, we have

$$
M^{-1} \int_{\Omega_{\rho}(x)}|\nabla u|^{2} d y \leq \int_{\Omega_{\rho}(x)} a_{\alpha \beta}^{i j} \frac{\partial u^{\beta}}{\partial y_{j}} \frac{\partial u^{\alpha}}{\partial y_{i}}+\rho^{-2}|u-c|^{2} d y .
$$

In addition, we assume a global coercivity condition on $D$ and the form $A$

$$
M^{-1} \int_{\Omega}|\nabla u|^{2} d y \leq A(u, u), \quad u \in \dot{W}_{D}^{1,2}(\Omega) .
$$

The conditions (2.6) and (2.7) are immediate if $D$ has non-empty interior and the coefficients satisfy the strong ellipticity condition

$$
a_{\alpha \beta}^{i j} \xi_{j}^{\beta} \xi_{i}^{\alpha} \geq c|\xi|^{2}, \quad \xi \in \mathbf{R}^{2 m} .
$$

At the end of this section, we will show that they also hold for the Lamé system when the set $D$ is non-empty and satisfies the corkscrew condition. 
We are now ready to give the precise formulation of (1.1). Given $f$ in $\dot{W}_{D}^{-1,2}(\Omega)$ and $f_{N} \in \dot{W}_{D}^{-1 / 2,2}(\partial \Omega)$ we say that $u$ is a weak solution of the mixed problem (1.1) if we have

$$
\left\{\begin{array}{l}
A(u, \phi)=-\langle f, \phi\rangle+\left\langle f_{N}, \phi\right\rangle_{\partial \Omega}, \quad \phi \in \dot{W}_{D}^{1,2}(\Omega) \\
u \in \dot{W}_{D}^{1,2}(\Omega) .
\end{array}\right.
$$

With our continuity (2.5) and global coercivity (2.7) assumptions, the existence and uniqueness of solutions is an immediate consequence of the Lax-Milgram theorem.

We also consider the adjoint problem for the operator $L^{*}$ whose coefficients are obtained by replacing $a_{\alpha \beta}^{i j}$ by $a_{\beta \alpha}^{j i}$. We say that $w$ is a weak solution of the mixed problem for $L^{*}$

$$
\begin{cases}L^{*} w=g, & \text { in } \Omega \\ \frac{\partial w}{\partial \nu}=g_{N}, & \text { on } N \\ w=0, & \text { on } D\end{cases}
$$

if we have

$$
\left\{\begin{array}{l}
A(\phi, w)=-\langle g, \phi\rangle+\left\langle g_{N}, \phi\right\rangle_{\partial \Omega}, \quad \phi \in \dot{W}_{D}^{1,2}(\Omega) \\
w \in \dot{W}_{D}^{1,2}(\Omega) .
\end{array}\right.
$$

Given a locally integrable function on $\Omega, x \in \bar{\Omega}$, a subset $D \subset \partial \Omega$, and $\rho \in\left(0, r_{0}\right)$, we define

$$
\bar{u}_{x, \rho}= \begin{cases}0, & \text { if } \operatorname{dist}\left(\Omega_{\rho}(x), D\right)=0 \\ f_{\Omega_{\rho}(x)} u(y) d y, & \text { if } \operatorname{dist}\left(\Omega_{\rho}(x), D\right)>0\end{cases}
$$

Here, we are using $f_{E} f d y=|E|^{-1} \int_{E} f d y$ to denote the average of $E$. We will define the space $B M O_{D}(\Omega)$ to be the collection of integrable functions $u$ on $\Omega$ for which the norm

$$
\|u\|_{*, D}=\sup \left\{f_{\Omega_{\rho}(x)}\left|u-\bar{u}_{x, \rho}\right| d y: x \in \bar{\Omega}, \rho \in\left(0, r_{0}\right)\right\}
$$

is finite. The supremum is taken over all local domains. We will be primarily interested in the case when $D \neq \emptyset$ and then it is easy to see that the above expression is a norm. In the case where $\Omega$ is $\mathbf{R}^{2}$ or $D=\emptyset$, then the elements of the space will be equivalence classes of functions which differ by a constant.

We note that if $D_{1} \subset D_{2}$, then we have that $B M O_{D_{1}}(\Omega) \supset B M O_{D_{2}}(\Omega)$ and in particular we have $B M O_{D}(\Omega) \subset B M O_{\emptyset}(\Omega)$. Thus, we obtain the JohnNirenberg inequality

$$
\left.\left|\left\{y \in \Omega_{\rho}(x):\left|u(y)-\bar{u}_{x, \rho}\right|>\lambda\right\}\right| \leq C \rho^{2} \exp \left(-\lambda /\|u\|_{*, D}\right)\right\} .
$$

The standard proof for $B M O\left(\mathbf{R}^{n}\right)$ as found in the lecture notes of Journé [15. Chapter 3], for example, extends easily to the space $B M O_{D}(\Omega)$. As a 
consequence, it follows that if $D \neq \emptyset$, then $B M O_{D}(\Omega) \subset L^{p}(\Omega)$, for $1 \leq p<$ $\infty$. In the case where $\Omega=\mathbf{R}^{2}$, we can show that any representative of a $B M O\left(\mathbf{R}^{2}\right)$ function lies in $\cap_{p<\infty} L_{l o c}^{p}\left(\mathbf{R}^{2}\right)$ and in the case of a bounded domain any representative of a function in $B M O_{\emptyset}(\Omega)$ also lies in $\cap_{p<\infty} L^{p}(\Omega)$.

We define atoms for $\Omega$ and $D$ and then the Hardy space $H_{D}^{1}(\Omega)$. We say that a bounded measurable function $a$ is an atom for $\Omega$ and $D$ if $a$ is supported in one of the local domains $\Omega_{\rho}(x)$ and satisfies

$$
\begin{aligned}
\|a\|_{L^{\infty}(\Omega)} & \leq 1 /\left|\Omega_{\rho}(x)\right| \\
\bar{a}_{x, \rho} & =0 .
\end{aligned}
$$

A function $f$ is in the atomic Hardy space $H_{D}^{1}(\Omega)$ if there is a sequence of atoms $\left\{a_{i}\right\}_{i=1}^{\infty}$ and a sequence of real numbers $\left\{\lambda_{i}\right\} \in \ell^{1}$ so that $f=\sum_{i=1}^{\infty} \lambda_{i} a_{i}$. We define a norm on this space by

$$
\|f\|_{H_{D}^{1}(\Omega)}=\inf \sum_{i=1}^{\infty}\left|\lambda_{i}\right|
$$

where the infinum is taken over all representations of $f$.

It will be useful to observe that the expression

$$
\sup \left\{\int_{\Omega} a^{\alpha} u^{\alpha} d y: a \text { is an atom for } \Omega \text { and } D\right\}
$$

gives an equivalent norm on $B M O_{D}(\Omega)$. This proposition may be found in Journé [15, Chapter 3] for $B M O\left(\mathbf{R}^{n}\right)$. The extension to $B M O_{D}(\Omega)$ is straightforward. We will use the characterization of the $B M O_{D}(\Omega)$ norm in (2.10) to show that the Green function lies in $B M O_{D}(\Omega)$. In fact, $B M O_{D}(\Omega)$ may be identified with the dual of the atomic Hardy space $H_{D}^{1}(\Omega)$. See Journé [15, Coifman and Weiss [6] for the case when $D=\emptyset$. Chang [3] treats the extreme cases where $D=\emptyset$ or $D=\partial \Omega$. The extension to general $D$ is not difficult.

We recall several Poincaré and Sobolev inequalities that will be needed in the argument below. The first inequality is a scale invariant Sobolev-Poincaré inequality. If $u \in \dot{W}_{D}^{1, p}(\Omega), 1 \leq p<2$ and $1 / q=1 / p-1 / 2$, and $\Omega_{\rho}(x)$ is one of the local domains defined above, then we may find a constant $C$ depending only on $M$ and $p$ so that

$$
\left(\int_{\Omega_{\rho}(x)}\left|u-\bar{u}_{x, \rho}\right|^{q} d y\right)^{1 / q} \leq C\left(\int_{\Omega_{2 \rho}(x)}|\nabla u|^{p} d y\right)^{1 / p}
$$

If $\bar{u}_{x, \rho}=f_{\Omega_{\rho}(x)} u d y$, the inequality holds with $\Omega_{\rho}(x)$ as the domain of integration on the right-hand side. However, if $\operatorname{dist}\left(\Omega_{\rho}(x), D\right)=0$, then we need to expand $\Omega_{\rho}(x)$ and use the corkscrew condition (2.1) in order to conclude that $u$ vanishes on a large enough set to obtain the inequality (2.11). See [23, Section 3] for more details. A useful consequence of the inequality (2.11) is the Poincaré inequality for $1 \leq p<\infty$,

$$
\left(\int_{\Omega_{\rho}(x)}\left|u-\bar{u}_{x, \rho}\right|^{p} d y\right)^{1 / p} \leq C \rho\left(\int_{\Omega_{2 \rho}(x)}|\nabla u|^{p} d y\right)^{1 / p}
$$


Note that the Poincaré inequality (2.12) with $p=2$ immediately implies the embedding $\dot{W}_{D}^{1,2}(\Omega) \subset B M O_{D}(\Omega)$. The corkscrew condition is needed to establish this embedding. If $2<p<\infty$, we have the following version of Morrey's inequality

$$
\left\|u-\bar{u}_{x, \rho}\right\|_{L^{\infty}\left(\Omega_{\rho}(x)\right)} \leq C \rho^{1-2 / p}\left(\int_{\Omega_{2 \rho}(x)}|\nabla u|^{p} d y\right)^{1 / p}
$$

This inequality gives the embedding of the Sobolev space $\dot{W}_{D}^{1, t}(\Omega)$ into space of Hölder continuous functions with exponent $1-2 / t$ when $t>2$. Finally, we give an estimate at the boundary. Let $\Omega_{\rho}(x)$ be one of our local domains and suppose that $1 \leq p<\infty, 1 \leq q<2$ and $1 / q=1 /(2 p)+1 / 2$. There exists a constant $C=C(p, M)$ so that for $u \in \dot{W}_{D}^{1, q}\left(\Omega_{2 \rho}(x)\right)$, we have

$$
\left(\int_{\Delta_{\rho}(x)}\left|u-\bar{u}_{x, \rho}\right|^{p} d y\right)^{1 / p} \leq C\left(\int_{\Omega_{2 \rho}(x)}|\nabla u|^{q} d y\right)^{1 / q}
$$

To end this section, we recall the Lamé operator and show that the form for this operator with the mixed boundary condition satisfies the ellipticity condition (2.6) and coercivity condition (2.7). Given two real-valued functions $\mu$ and $\lambda$, the Lamé operator is the operator with coefficients

$$
a_{\alpha \beta}^{i j}(x)=\mu(x)\left(\delta_{i j} \delta_{\alpha \beta}+\delta_{j \alpha} \delta_{i \beta}\right)+\lambda(x) \delta_{i \alpha} \delta_{j \beta}
$$

We will use $\epsilon(u)$ to denote the strain or the symmetric part of the gradient,

$$
\epsilon_{\alpha}^{i}(u)=\frac{1}{2}\left(\frac{\partial u^{i}}{\partial x_{\alpha}}+\frac{\partial u^{\alpha}}{\partial x_{i}}\right)
$$

and then $\sigma(u)$ will denote the stress tensor which is given by

$$
\sigma_{\alpha}^{i}(u)=a_{\alpha \beta}^{i j} \frac{\partial u^{\beta}}{\partial x_{j}}
$$

or more compactly by

$$
\sigma(u)=2 \mu \epsilon(u)+\lambda I \operatorname{div} u
$$

where $I$ is the $2 \times 2$ identity matrix. The functions $\mu$ and $\lambda$ are called the Lamé parameters and are related to the elastic properties of the material. We require that $\lambda$ and $\mu$ are bounded, measurable functions and that $\mu(x)-\lambda^{-}(x) \geq c>0$ where $\lambda^{-}$denotes the negative part of the function $\lambda$. With this assumption, we have the pointwise lower bound

$$
\sigma_{\alpha}^{i} \frac{\partial u^{\alpha}}{\partial x_{i}} \geq c|\epsilon(u)|^{2}
$$


We recall the following version of Korn's second inequality. Let $\Omega_{\rho}(x)$ be one of our local domains with star-center $x^{*}$. If $u$ is in $\dot{W}^{1,2}\left(\Omega_{\rho}(x)\right)$ and $c$ is any constant vector in $\mathbf{R}^{2}$, then

$$
\int_{\Omega_{\rho}(x)}|\nabla u|^{2} d y \leq C\left(\int_{\Omega_{\rho}(x)}|\epsilon(u)|^{2} d y+\frac{1}{\rho^{2}} \int_{B_{\rho / 2}\left(x^{*}\right)}|u-c|^{2} d y\right)
$$

The constant depends only on $M$. This may be established using the argument given in the monograph of Oleı̆nik, Shamaev, and Yosifian [22, Theorem 2.10]. It is clear that the ellipticity condition (2.6) follows from the pointwise bound (2.15) and (2.16). If $D$ is non-empty (and then the corkscrew condition implies $D$ has non-empty interior), the coercivity condition (2.7) may also be found in Oleünik, Shamaev, and Yosifian [22, Theorem 2.7], however the standard proof of this inequality seems to be by contradiction and thus we cannot say anything about how the constant depends on the domain $\Omega$ and the boundary set $D$.

All of our quantitative assumptions have been in terms of the constant $M$. The results below will be of two types. Many of the results will be local estimates which hold on scales $\rho \in\left(0, r_{0}\right)$. In these local estimates, the constant will depend only on $m, M$, and any $L^{p}$-indices that appear in the estimate. The remaining estimates will depend on global properties of the domains such as the collection of coordinate cylinders which cover the boundary or the constant in the Poincaré inequality (2.2). However, the constant may be chosen to be uniform under small changes in the Lipschitz functions which define the boundary. One exception is the constant in the Korn inequality which gives the coercivity condition (2.7) for the Lamé system. As noted above, the standard proof is by contradiction and gives no information about the dependence of the constant on the domain. In the study of the Neumann problem the global estimates for solutions will depend on the estimate in our existence theorem, Theorem 5.4. The proof of this result uses the Fredholm theory and thus we have no information about the behavior of the operators used to solve the Neumann problem.

\section{Estimates for solutions of the mixed prob- lem}

In this section, we prove estimates for solutions of the mixed problem. We will use the reverse Hölder technique of Giaquinta and Modica [11]. Before beginning the main argument, we introduce two auxiliary functions which will arise when we prove Caccioppoli-type inequalities for solutions of the mixed problem (2.8) when the data $f$ or $f_{N}$ is not zero.

In the following definitions, we assume that $0<r<r_{0}$ so that we can define $\Omega_{\rho}(x)$ and $\Delta_{\rho}(x)$ for $0<\rho<r$. For $0<\alpha<2$, we let $I_{\alpha, r} f(x)$ denote the maximal fractional integral given by

$$
I_{\alpha, r} f(x)=\sup _{0<\rho<r} \rho^{\alpha-2} \int_{\Omega_{\rho}(x)}|f(y)| d y .
$$


Note that we have several choices for $\Omega_{\rho}(x)$ when $x$ lies in several coordinate rectangles. In defining $I_{\alpha, r}$, we take the maximum over the choices for $\Omega_{\rho}(x)$ arising from our finite cover of the boundary by coordinate rectangles. We have that $\rho^{\alpha-2} \chi_{\Omega_{\rho}(x)}(y) \leq C(M)|x-y|^{\alpha-2}$ and it follows that $I_{\alpha, r} f(x) \leq C R_{\alpha}|f|(x)$ where $R_{\alpha}$ is the standard Riesz potential. Thus, the Hardy-Littlewood-Sobolev theorem gives us that with $q$ and $p$ related by $1 / q=1 / p+\alpha / 2$ and $2 /(2-\alpha)<$ $p<\infty$, we have

$$
\left\|I_{\alpha, r} f\right\|_{L^{p}(\Omega)} \leq C(p, M)\|f\|_{L^{q}(\Omega)} .
$$

Next, if $f_{N}$ is a function on $\partial \Omega$, we define

$$
P_{r} f_{N}(x)=\sup \left\{f_{\Delta_{\rho}(\hat{x})}\left|f_{N}\right| d \sigma: \operatorname{dist}(x, \partial \Omega)<\rho<r\right\}
$$

with the convention that the supremum of the empty set is zero. Recall that if $x$ is in a coordinate rectangle $Z_{r}(y)$, and $x=\left(x_{1}, \phi\left(x_{1}\right)+t e_{2}\right)$, we defined $\hat{x}=\left(x_{1}, \phi\left(x_{1}\right)\right)$. We also note that if $\operatorname{dist}(x, \partial \Omega) \leq \rho$, then $\Delta_{\rho}(\hat{x})=\partial \Omega \cap \partial \Omega_{\rho}(x)$ and if $\operatorname{dist}(x, \partial \Omega)>\rho$, then $\partial \Omega \cap \partial \Omega_{\rho}(x)=\emptyset$. Our Neumann data, $f_{N}$ is initially only defined on $N$. In the definition of $P_{r}$, we assume that $f_{N}$ has been extended to $\partial \Omega$ by setting $f_{N}=0$ outside $N$. Following the argument in [23, Section 3], for $1<p<\infty$, we may find a constant $C=C(M, p, \Omega)$ so that we have the estimate

$$
\left(\int_{\Omega}\left|P_{r} f\right|^{2 p} d y\right)^{1 / 2 p} \leq C\left(\int_{\partial \Omega}|f|^{p} d \sigma\right)^{1 / p}
$$

and the corresponding estimate for $p=\infty$ is trivial.

We are now ready to begin our estimates. We begin with a simple energy estimate.

Proposition 3.3 If $f$ lies in the Hardy space $H_{D}^{1}(\Omega)$ and $f_{N}=0$, then the weak mixed problem (2.8) has a unique solution $u$ which satisfies

$$
\int_{\Omega}|\nabla u|^{2} d y \leq C\|f\|_{H_{D}^{1}(\Omega)}^{2}
$$

Proof. Let $a$ be an atom for the Hardy space $H_{D}^{1}(\Omega)$. Using the Poincaré inequality (2.12), it is easy to see that the map $u \rightarrow \int_{\Omega} a^{\alpha} u^{\alpha} d y$ lies in the dual of $\dot{W}_{D}^{1,2}(\Omega)$. As a consequence, the solution of the mixed problem (2.8) with $f=a$ and $f_{N}=0$ will satisfy $\int_{\Omega}|\nabla u|^{2} d y \leq C$. The result for a general element of $H_{D}^{1}(\Omega)$ follows easily from the result for an atom.

The following theorem gives our main estimate for solutions of the mixed boundary value problem. We note that the estimates of this theorem hold for the extreme cases where $D=\emptyset$ or $\partial \Omega$. In the arguments below, we will use the standard notation $1 / p^{\prime}=1-1 / p$ to denote the conjugate exponent.

Theorem 3.4 Let $\Omega$ be a Lipschitz domain, let $D \subset \partial \Omega$ be a (possibly empty) set that satisfies the corkscrew condition (2.1), and suppose that $L$ is an elliptic 
operator which satisfies the conditions (2.4) and (2.6). Let $u$ be a weak solution of the mixed problem (2.8) and assume that $f \in L^{p}(\Omega)$ and $f_{N} \in L^{p_{N}}(N)$ are functions. We may find a $t_{0}>2$ so that with $t \in\left[2, t_{0}\right), p \in(1,2)$ and $p_{N} \in(1, \infty), x \in \bar{\Omega}$ and $\rho \in\left(0, r_{0}\right)$ we have the estimate

$$
\begin{aligned}
\left(f_{\Omega_{\rho}(x)}|\nabla u|^{t} d y\right)^{1 / t} \leq C & {\left[\left(f_{\Omega_{2 \rho}(x)}|\nabla u|^{2} d y\right)^{1 / 2}+\left(f_{\Omega_{2 \rho}(x)} I_{p, 2 \rho}\left(|f|^{p}\right)^{t / p} d y\right)^{1 / t}\right.} \\
& \left.+\left(f_{\Omega_{2 \rho}(x)} P_{2 \rho}\left(\left|f_{N}\right|^{p_{N}}\right)^{t / p_{N}} d y\right)^{1 / t}\right] .
\end{aligned}
$$

The exponent $t_{0}$ depends on $M, p$, and $p_{N}$ and the constant $C=C\left(M, t, p, p_{N}\right)$.

Proof. We let $u$ be a weak solution of the mixed problem (2.8) with $f \in L^{p}(\Omega)$ and $f_{N} \in L^{p_{N}}(N)$ for some $p>1$ and $p_{N}>1$. Define $q$ by $1 / q=3 / 2-1 / p$ and $q_{N}$ by $1 / q_{N}=1-1 /\left(2 p_{N}\right)$ and let $s=\max \left(q, q_{N}\right)<2$. We fix $x \in \bar{\Omega}$, $0<\rho<r_{0}$ and we claim the Caccioppoli inequality

$$
\begin{aligned}
& f_{\Omega_{\rho}(x)}|\nabla u|^{2} d y \\
& \leq C\left[\left(f_{\Omega_{4 \rho}(x)}|\nabla u|^{s} d y\right)^{2 / s}+I_{p, 2 \rho}\left(|f|^{p}\right)^{2 / p}(x)+P_{2 \rho}\left(\left|f_{N}\right|^{p_{N}}\right)^{2 / p_{N}}(x)\right]
\end{aligned}
$$

The estimate of the theorem follows immediately from (3.5) and the reverse Hölder argument of Giaquinta and Modica (see [10, Theorem 1.2, p. 122]). To establish (3.5), we fix $x \in \bar{\Omega}$ and $\rho \in\left(0, r_{0}\right)$. We let $\eta$ be a cutoff function which is one on $\Omega_{\rho}(x)$, zero on $\Omega \backslash \Omega_{2 \rho}(x)$, and satisfies $|\nabla \eta| \leq C / \rho$. We let $E=\int_{\Omega_{2 \rho}(x)}\left|u-\bar{u}_{x, 2 \rho}\right| \eta|\nabla \eta||\nabla u|+\rho^{-2}\left|u-\bar{u}_{x, 2 \rho}\right|^{2} d y$. As our first step, we will establish the inequality

$$
\begin{aligned}
& \frac{1}{\rho^{2}} \int_{\Omega_{2 \rho}(x)} \eta^{2}|\nabla u|^{2} d y \leq C\left(\frac{1}{\rho^{2}} E+\left(f_{\Omega_{4 \rho}(x)}|\nabla u|^{s} d y\right)^{2 / s}\right. \\
& \left.+\left(\frac{1}{\rho^{2-p}} \int_{\Omega_{2 \rho}(x)}|f|^{p} d y\right)^{2 / p}+\left(f_{\partial \Omega \cap \Omega_{2 \rho}(x)}\left|f_{N}\right|^{p_{N}} d \sigma\right)^{2 / p_{N}}\right)
\end{aligned}
$$

If we use (2.11) and the Cauchy inequality with $\epsilon$ 's, the estimate (3.6) implies (3.5). Thus we turn to the proof of (3.6).

To establish (3.6), observe that thanks to our definition of $\bar{u}_{x, \rho}$, we have $v=\eta^{2}\left(u-\bar{u}_{x, 2 \rho}\right) \in \dot{W}_{D}^{1,2}(\Omega)$ whenever $u \in \dot{W}_{D}^{1,2}(\Omega)$. Thus, from the product rule, our ellipticity assumption and the weak formulation of the mixed problem, 
(2.8), we obtain

$$
\begin{aligned}
\int_{\Omega} \eta^{2}|\nabla u|^{2} d y & \leq \int_{\Omega}\left|\nabla\left(\eta\left(u-\bar{u}_{x, 2 \rho}\right)\right)\right|^{2} d y+C E \\
& \leq C\left(A\left(\eta\left(u-\bar{u}_{x, 2 \rho}\right), \eta\left(u-\bar{u}_{x, 2 \rho}\right)\right)+E\right) \\
& \leq C(A(u, v)+E) \\
& \leq C\left(\int_{\partial \Omega} f_{N}^{\alpha} v^{\alpha} d \sigma-\int_{\Omega} f^{\alpha} v^{\alpha} d y+E\right)
\end{aligned}
$$

We claim that if $1<p<2$, we have

$$
\left|\frac{1}{\rho^{2}} \int_{\Omega} f^{\alpha} v^{\alpha} d y\right| \leq\left(f_{\Omega_{2 \rho}(x)}|\nabla u|^{q} d y\right)^{2 / q}+C\left(\frac{1}{\rho^{2-p}} \int_{\Omega_{2 \rho}(x)}|f|^{p} d y\right)^{2 / p} .
$$

We also will need that if $1<p_{N} \leq \infty$, then

$$
\begin{aligned}
& \left|\frac{1}{\rho^{2}} \int_{N} f_{N}^{\alpha} v^{\alpha} d \sigma\right| \\
& \quad \leq\left(f_{\Omega_{2 \rho}(x)}|\nabla u|^{q_{N}} d y\right)^{2 / q_{N}}+C\left(f_{\partial \Omega_{\cap} \Omega_{2 \rho}(x)}\left|f_{N}\right|^{p_{N}} d \sigma\right)^{2 / p_{N}} .
\end{aligned}
$$

It is easy to see that (3.6) follows from (3.7-3.9) and thus it remains to prove (3.8) and (3.9).

To establish (3.8), we use Hölder's inequality with $1<p<2$ and then the Sobolev-Poincaré inequality (2.11) to obtain that with $1 / q=3 / 2-1 / p$

$$
\begin{aligned}
\left|\frac{1}{\rho^{2}} \int_{\Omega} f^{\alpha} v^{\alpha} d y\right| & \leq \frac{1}{\rho^{2}}\left(\int_{\Omega_{2 \rho}(x)}|f|^{p} d y\right)^{1 / p}\left(\int_{\Omega_{2 \rho}(x)}\left|u-\bar{u}_{x, 2 \rho}\right|^{p^{\prime}} d y\right)^{1 / p^{\prime}} \\
& \leq \frac{C}{\rho^{2 / q-1+2 / p}}\left(\int_{\Omega_{2 \rho}(x)}|f|^{p} d y\right)^{1 / p}\left(\int_{\Omega_{4 \rho}(x)}|\nabla u|^{q} d y\right)^{1 / q} .
\end{aligned}
$$

Now (3.8) follows easily.

To establish (3.9), we use Hölder's inequality and the boundary Poincaré inequality (2.14) to obtain with $1<p_{N}<\infty$ and $1 / q_{N}=1-1 /\left(2 p_{N}\right)$, that

$$
\begin{aligned}
& \left|\frac{1}{\rho^{2}} \int_{N} f_{N}^{\alpha} v^{\alpha} d \sigma\right| \\
& \quad \leq \frac{1}{\rho^{2}}\left(\int_{\partial \Omega \cap \partial \Omega_{2 \rho}(x)}\left|f_{N}\right|^{p_{N}} d \sigma\right)^{1 / p_{N}}\left(\int_{\partial \Omega \cap \partial \Omega_{2 \rho}(x)}\left|u-\bar{u}_{x, 2 \rho}\right|^{p_{N}^{\prime}} d y\right)^{1 / p_{N}^{\prime}} \\
& \quad \leq \frac{C}{\rho^{1 / p_{N}+2 / q_{N}}}\left(\int_{\partial \Omega \cap \partial \Omega_{2 \rho}(x)}\left|f_{N}\right|^{p_{N}} d \sigma\right)^{1 / p_{N}}\left(\int_{\Omega_{4 \rho}(x)}|\nabla u|^{q_{N}} d y\right)^{1 / q_{N}}
\end{aligned}
$$

and now (3.9) follows. 
Remark. The argument above also gives us a Caccioppoli inequality for solutions. Let $u$ be a solution of (2.8) with $f=0$ and $f_{N} \in L^{2}(N)$, fix $x$ and $\rho$ and let $v$ be as in (3.7). Since $v$ vanishes outside $\Omega_{2 \rho}(x)$, we may use (2.14) and Hölder's inequality to obtain $\int_{\partial \Omega}|v|^{2} d \sigma \leq C \rho \int_{\Omega}|\nabla v|^{2} d y$. If $\operatorname{dist}(x, \partial \Omega)>2 \rho$, the boundary term in (3.7) is zero. When $\operatorname{dist}(x, \partial \Omega)<2 \rho$, we estimate the boundary term in (3.7) using the Cauchy inequality with $\epsilon$ 's and the above observation to obtain

$$
\left|\int_{N} f_{N}^{\alpha} v^{\alpha} d \sigma\right| \leq C\left(\frac{\rho}{\epsilon} \int_{\Delta_{2 \rho}(x)}\left|f_{N}\right|^{2} d \sigma+E\right)+\epsilon \int_{\Omega} \eta^{4}|\nabla u|^{2} d y
$$

Using this estimate in (3.7) and the Cauchy inequality with $\epsilon$ 's gives

$$
\int_{\Omega_{\rho}(x)}|\nabla u|^{2} d y \leq C\left(\frac{1}{\rho^{2}} \int_{\Omega_{2 \rho}(x)}\left|u-\bar{u}_{x, 2 \rho}\right|^{2} d y+\rho \int_{\partial \Omega \cap \partial \Omega_{2 \rho}(x)} f_{N}^{2} d \sigma\right) .
$$

Corollary 3.11 Let $\Omega, D$, and $L$ be as in Theorem 3.4. Let $u$ be a solution of the weak mixed problem (2.8) with $f=0$ on $\Omega_{2 \rho}(x)$ and $f_{N} \in L^{2}(\partial \Omega)$. There is a constant $C$ and $\gamma_{0}>0$ so that for $\gamma$ in $\left(0, \gamma_{0}\right)$, we have

$$
\begin{array}{r}
|u(y)-u(z)| \leq C \frac{|y-z|^{\gamma}}{\rho^{\gamma}}\left(\left(f_{\Omega_{2 \rho}(x)}\left|u-\bar{u}_{x, 2 \rho}\right|^{2} d y\right)^{1 / 2}\right. \\
\left.+\left(\rho \int_{\partial \Omega \cap \partial \Omega_{2 \rho}(x)}\left|f_{N}\right|^{2} d \sigma\right)^{1 / 2}\right), y, z \in \Omega_{\rho}(x) \\
|u(x)| \leq C\left(\left(f_{\Omega_{2 \rho}(x)}|u|^{2} d y\right)^{1 / 2}+\left(\rho \int_{\partial \Omega \cap \partial \Omega_{2 \rho}(x)}\left|f_{N}\right|^{2} d \sigma\right)^{1 / 2}\right)
\end{array}
$$

The constant $\gamma_{0}$ depends only on $M$ and $C$ depends on $M$ and $\gamma$.

Proof. We let $u$ be a solution of (2.8) with $f=0$ in $\Omega_{2 \rho}(x)$. We observe that there is a constant $c$ so that if $y \in \Omega_{3 \rho / 2}(x), 0<s<c \rho$, then $\Omega_{2 s}(y) \subset \Omega_{2 \rho}(x)$ and we have $I_{\alpha, 2 s} f=0$. Thus if $\Omega_{s}(y) \subset \Omega_{\rho}(x)$ and $s<c \rho$, the main estimate of Theorem 3.4 and (3.1) imply that for $t<\min \left(4, t_{0}\right)$

$$
\left(f_{\Omega_{s}(y)}|\nabla u|^{t} d y\right)^{1 / t} \leq C\left(\left(f_{\Omega_{2 s}(y)}|\nabla u|^{2} d y\right)^{1 / 2}+\frac{\rho}{s}\left(f_{\partial \Omega \cap \partial \Omega_{2 \rho}(x)}\left|f_{N}\right|^{2} d \sigma\right)^{1 / 2}\right) .
$$

Using (3.14) with the Caccioppoli inequality (3.10) and Morrey's inequality (2.13), we obtain the Hölder estimate (3.12) with $\gamma_{0}=\min \left(1-2 / t_{0}, 1 / 2\right)$ where $t_{0}$ is as in Theorem 3.4.

To obtain the estimate (3.13), we write

$$
|u(x)| \leq\left|u(x)-\bar{u}_{x, \rho}\right|+\left|\bar{u}_{x, \rho}\right|,
$$

use the Hölder estimate (3.12) to bound the first term on the right and then the Hölder inequality. Note that this works even when $\bar{u}_{x, \rho}=0$ since in this case, $u$ vanishes on a nearby piece of the boundary. 
We observe a version of the Green identity for solutions of the weak mixed problem (2.8) and the corresponding problem for the adjoint operator, $L^{*}$. If $u$ satisfies $L u=f$ with $\partial u / \partial \nu=f_{N}$ and $w$ is a solution of the adjoint problem $L^{*} w=g$ with $\partial w / \partial \nu=g_{N}$ and suppose that $f$ and $g$ are in $L^{p}(\Omega)$ and $f_{N}$ and $g_{N}$ are in $L^{p}(\partial \Omega)$ for some $p>1$. Then, we have

$$
\int_{\Omega} u^{\alpha} g^{\alpha} d y-\int_{N} u^{\alpha} g_{N}^{\alpha} d \sigma=\int_{\Omega} w^{\alpha} f^{\alpha} d y-\int_{N} w^{\alpha} f_{N}^{\alpha} d \sigma
$$

The identity (3.15) follows easily since both sides of (3.15) are equal to $A(u, v)$.

The following Corollary is only valid when $D \neq \emptyset$. For the Neumann problem, there are non-constant solutions to the homogeneous problem and hence the estimates (3.17) and (3.18) cannot hold. We will give a version of this result for the Neumann problem in section 5 .

Corollary 3.16 Suppose that $D$ is a non-empty subset of $\partial \Omega$ that satisfies the corkscrew condition. Let $L, \Omega$, and $t_{0}$ be as in Theorem 3.4, suppose that $t \in\left(2, t_{0}\right)$, define $r$ by $1 / r=1 / 2+1 / t$ and let $r_{N}=t / 2$. If $u$ is a weak solution of the mixed problem (2.8) with $f_{N}=0$ and $f \in L^{r}(\Omega)$, then we have

$$
\|\nabla u\|_{L^{t}(\Omega)} \leq C\|f\|_{L^{r}(\Omega)} .
$$

If $u$ is a solution of (2.8) with $f \in \dot{W}_{D}^{-1, t^{\prime}}(\Omega)$ and $f_{N}=0$, then we have the estimate

$$
\|u\|_{L^{r^{\prime}}(\Omega)} \leq C\|f\|_{\dot{W}_{D}^{-1, t^{\prime}}(\Omega)} .
$$

The constant $C$ depends on $M, t$, and $\Omega$.

Proof. Using the estimate (2.2), it follows that the map $v \rightarrow \lambda(v)=\int_{\Omega} f^{\alpha} v^{\alpha} d y$ is an element in $\dot{W}_{D}^{-1,2}(\Omega)$ with the bound $\|\lambda\|_{\dot{W}_{D}^{-1,2}(\Omega)} \leq C r_{0}^{2 / r^{\prime}}\|f\|_{L^{r}(\Omega)}$. As a consequence, if $u$ is a weak solution of the mixed problem (2.8) with $f \in L^{r}(\Omega)$, $r>1$ and $f_{N}=0$, we have the energy estimate

$$
\|\nabla u\|_{L^{2}(\Omega)} \leq C r_{0}^{2 / r^{\prime}}\|f\|_{L^{r}(\Omega)} .
$$

We begin with the main estimate of Theorem 3.4 on domains $\Omega_{r_{0}}(x)$. We choose $p$ with $p \in(1, r)$ and apply the estimate (3.1) to obtain

$$
\left(\int_{\Omega_{r_{0}}(x)}|\nabla u|^{t} d y\right)^{1 / t} \leq C\left(r_{0}^{2 / t-1}\left(\int_{\Omega_{2 r_{0}(x)}}|\nabla u|^{2} d y\right)^{1 / 2}+\|f\|_{L^{r}(\Omega)}\right) .
$$

We use the energy estimate (3.19) and observe that $1-2 / t=2 / r^{\prime}$. Finally, we cover $\Omega$ with domains $\Omega_{r_{0}}(x)$ and use Minkowski's inequality to obtain the first estimate (3.17).

The second estimate (3.18) for solutions of $L$ follows by duality from (3.15) and the first estimate (3.17) for solutions of the adjoint operator $L^{*}$. If $A^{*}$ is the form for $L^{*}$, then $A^{*}(u, v)=A(v, u)$, thus it is clear that $L^{*}$ satisfies the hypotheses of this theorem exactly when $L$ does. 
Our next estimates are valid only when $D \neq \emptyset$. The estimates may fail for the Neumann problem due to non-uniqueness.

Corollary 3.20 Suppose that $D \neq \emptyset$ and satisfies the corkscrew condition. If $u$ is a solution of the weak mixed problem (2.8) with $f$ an atom and $f_{N}=0$, then we have

$$
\|u\|_{L^{\infty}(\Omega)} \leq C
$$

Proof. We let $u$ be a solution of the weak mixed problem with $f=a$, an atom for the Hardy space $H_{D}^{1}(\Omega)$, and $f_{N}=0$. We suppose that $a$ is supported in $\Omega_{\rho}(x)$.

To estimate $u$, we fix $z$ in $\Omega, t>2$, and use the Morrey inequality (2.13) and the Hölder inequality to obtain

$$
\begin{aligned}
|u(z)| & \leq\left|u(z)-\bar{u}_{z, \rho}\right|+\left|\bar{u}_{z, \rho}\right| \\
& \leq C\left(\rho^{1-2 / t}\|\nabla u\|_{L^{t}(\Omega)}+\rho^{2 / t-1}\|u\|_{L^{r^{\prime}(\Omega)}}\right) .
\end{aligned}
$$

As in Corollary 3.16, $r$ is defined by $1 / r=1 / 2+1 / t$ and $r^{\prime}$ is the dual exponent given by $1 / r^{\prime}=1 / 2-1 / t$.

We restrict $t$ to lie in the interval $\left(2, t_{0}\right)$ with $t_{0}$ as in Theorem 3.4 and show how to use Corollary 3.16 to estimate the two terms on the right-hand side of (3.21). First, we use (3.17) and the normalization of the atom to conclude that

$$
\|\nabla u\|_{L^{t}(\Omega)} \leq C\|a\|_{L^{r}(\Omega)} \leq C \rho^{2 / t-1} .
$$

Next, we claim that for $t>2$, we have

$$
\|a\|_{\dot{W}^{-1, t^{\prime}}(\Omega)} \leq C \rho^{1-2 / t} .
$$

Given the claim, the estimate (3.18) in Corollary 3.16 implies that we have

$$
\|u\|_{L^{r^{\prime}}(\Omega)} \leq C \rho^{1-2 / t} .
$$

To establish the claim (3.23), we use that either $\bar{v}_{x, \rho}=0$ or $\int_{\Omega} a d y=0$, the normalization of the atom, and the Morrey inequality (2.13) to write

$$
\left|\int_{\Omega} a^{\alpha} v^{\alpha} d y\right|=\left|\int_{\Omega} a^{\alpha}\left(v-\bar{v}_{x, \rho}\right)^{\alpha} d y\right| \leq C \rho^{1-2 / t}\|\nabla v\|_{L^{t}(\Omega)}
$$

which gives the claim (3.23).

The estimate of the Corollary follows from (3.21), (3.22), and (3.24).

\section{The Green function for the mixed prob- lem}

In this section, we prove the existence of the Green function for the mixed problem and give its main properties. We allow the case when $D=\partial \Omega$ which gives the Dirichlet problem. 
We begin by giving our definition of a Green function. This formulation is modeled on the definition in Littman, Stampacchia, and Weinberger [18. We say that $G(x, y)=\left(G^{\alpha \beta}(x, y)\right)_{\alpha, \beta=1, \ldots, m}$ is a Green function with pole at $x$ for the mixed problem (2.8) if $G(x, \cdot) \in L^{1}(\Omega)$ and whenever $u$ is the weak solution of the mixed problem with $f \in C(\bar{\Omega})$ and $f_{N}=0$, then we have

$$
u^{\alpha}(x)=\int_{\Omega} G^{\alpha \beta}(x, y) f^{\beta}(y) d y .
$$

If we have existence and uniqueness for the mixed problem (2.8) when the right-hand side, $f$ is in $C(\bar{\Omega})$, then it is obvious that the Green function is unique. The following theorem shows that the Green function exists and gives additional regularity of the Green function.

Theorem 4.1 Let $\Omega$ be a bounded Lipschitz domain in the plane and let $D \subset$ $\partial \Omega$ be a non-empty open set satisfying the interior corkscrew condition. If $L$ is an elliptic operator satisfying (2.4), (2.6), and (2.7) on $\dot{W}_{D}^{1,2}(\Omega)$. Then there exists a unique Green function $G(x, y)$ for the mixed problem which satisfies

$$
\begin{aligned}
& G(x, \cdot) \in B M O_{D}(\Omega), \quad x \in \Omega \\
& \nabla_{y} G(x, \cdot) \in L^{2}\left(\Omega \backslash \Omega_{r}(x)\right), \quad \text { for } x \in \Omega, r \in\left(0, r_{0}\right) \\
& |G(x, y)| \leq C(1+\log (d /|x-y|)), \quad x, y \in \Omega \\
& |G(x, y)-G(x, z)| \leq C \frac{|y-z|^{\gamma}}{|x-y|^{\gamma}}, \quad x, y, z \in \Omega,|y-z|<\frac{1}{2} \mid x-y(4.5) \\
& |G(x, y)| \leq C \frac{\operatorname{dist}(y, D)^{\gamma}}{|x-y|^{\gamma}}, \quad x, y \in \Omega, \operatorname{dist}(y, D)<\frac{1}{2}|x-y|
\end{aligned}
$$

In these estimates $d$ is the diameter of $\Omega$ and the constants depend on the global character of $\Omega$ as well as $M$.

If we let $G$ be the Green function for the mixed problem for $L$ and $\tilde{G}$ the Green function for $L^{*}$ then we have

$$
G^{\alpha \beta}(x, y)=\tilde{G}^{\beta \alpha}(y, x), \quad \alpha, \beta=1, \ldots, m .
$$

Furthermore, if $f \in L^{p}(\Omega)$ and $f_{N} \in L^{p}(N)$ for some $p>1$, then the unique solution of the weak mixed problem (2.8) is given by

$$
u^{\alpha}(x)=\int_{\Omega} G^{\alpha \beta}(x, y) f^{\beta}(y) d y-\int_{N} G^{\alpha \beta}(x, y) f_{N}^{\beta}(y) d \sigma .
$$

Proof. We fix $x \in \Omega, \alpha$ in $\{1, \ldots, m\}$, and $\rho \in\left(0, r_{0}\right)$. We let $G_{\rho}^{\alpha \cdot}(x, \cdot)$ be the solution of mixed problem (2.9) for $L^{*}$ with this $g=e_{\alpha} \chi_{\Omega_{\rho}(x)} /\left|\Omega_{\rho}(x)\right|$ and $g_{N}=0$. We fix $f$ in $L^{p}(\Omega)$ and $f_{N} \in L^{p_{N}}(N)$ with $p>1$ and $p_{N}>1$ and let $u$ be a weak solution of the mixed problem for $L(2.8)$ with data $f$ and $f_{N}$. If we let $w=G_{\rho}^{\alpha \cdot}(x, \cdot)$ in (3.15), we obtain

$$
f_{\Omega_{\rho}(x)} u^{\alpha}(y) d y=\int_{\Omega} G_{\rho}^{\alpha \beta}(x, y) f^{\beta}(y) d y-\int_{N} G_{\rho}^{\alpha \beta}(x, y) f_{N}^{\beta}(y) d \sigma .
$$


If $f_{N}$ is zero and $f$ is an atom for $H_{D}^{1}(\Omega)$, we may use the estimate of Corollary 3.20 and the equivalent norm on $B M O_{D}(\Omega)$ given in (2.10) to conclude that $\left\|G_{\rho}(x, \cdot)\right\|_{*, D} \leq C$ with the constant $C$ depending only on $M$ and the global properties of $\Omega$. The Banach-Alaoglu theorem gives that for each $x$, there is a sequence $\left\{\rho_{j}\right\}$ with $\lim _{j \rightarrow \infty} \rho_{j}=0$ and a function $G(x, \cdot) \in B M O_{D}(\Omega)$ so that $G_{\rho_{j}}(x, \cdot)$ converges to $G(x, \cdot)$ in the weak-* topology of $B M O_{D}(\Omega)$. Since $u$ is Hölder continuous, the left-hand side of (4.9) converges to $u^{\alpha}(x)$. Since $L^{p}(\Omega) \subset H_{D}^{1}(\Omega)$ for all $p>1$, we obtain the representation (4.8) in the case that $f_{N}=0$. This gives us that $G(x, \cdot)$ is a Green function for the mixed problem with pole at $x$. Thus, we have established that the Green function lies in $B M O_{D}(\Omega)$, (4.2).

If we choose any sequence $\left\{\rho_{k}\right\}$ with $\lim _{k \rightarrow \infty} \rho_{k}=0$, the above argument, applied to the rows of $G_{\rho_{k}}$, gives a subsequence of $\left\{G_{\rho_{k}}(x, \cdot)\right\}$ which converges to a Green function. As the Green function is unique, the limit must be the function $G(x, \cdot)$. This implies that the entire family $\left\{G_{\rho}(x, \cdot)\right\}_{\rho}$ converges to $G(x, \cdot)$ in the weak-* topology of $B M O_{D}(\Omega)$.

Next, we recall that $B M O_{D}(\Omega) \subset L^{p}(\Omega)$ for any $p<\infty$. Thus we may use the Caccioppoli inequality (3.10), to conclude that

$$
\int_{\Omega \backslash \Omega_{r}(x)}\left|\nabla_{y} G_{\rho}(x, y)\right|^{2} d y \leq C(r), \quad \rho<r / 2 .
$$

This estimate will also hold for the limit and thus we obtain the conclusion (4.3) and that the rows of $G(x, \cdot)$ are solutions of $L^{*} G^{\alpha \cdot}(x, \cdot)=0$ in $\Omega \backslash\{x\}$. More precisely, we have $A\left(\phi, G^{\alpha \cdot}(x, \cdot)\right)=0$ whenever $\phi \in \dot{W}_{D}^{1,2}(\Omega)$ and $\phi$ vanishes in a neighborhood of $x$. Since $G(x, \cdot)$ is a solution of the adjoint equation, we have the estimates of Corollary 3.11 in $\Omega \backslash\{x\}$.

We show how to use these estimates to obtain the pointwise bounds of the Theorem. If $x$ and $y$ are in $\Omega$, we may find a chain of domains $\Omega_{j}=\Omega_{\rho_{j}}\left(y_{j}\right)$ for $j=0, \ldots, N$ so that a) $\Omega_{j} \subset \Omega \backslash\{x\}, \Omega_{j} \cap \Omega_{j+1} \neq \emptyset$ for $j=0, \ldots, N-1$, b) $\rho_{0} \geq$ $c|x-y|, y_{0}=y, \rho_{N}=r_{0} / 2$, c) $1 \leq \rho_{j+1} / \rho_{j} \leq 2$, and d) $N \leq C \log (d /|x-y|)$. Since $G(x, \cdot)$ is in $B M O_{D}(\Omega)$, we have that

$$
\left|f_{\Omega_{j}} G(x, y) d y-f_{\Omega_{j+1}} G(x, y) d y\right| \leq C\|G(x, \cdot)\|_{*, D} .
$$

Since the rows of $G(x, \cdot)$ are solutions of the equation $L^{*} u=0$, the properties of the chain $\left\{\Omega_{j}\right\}$ and the bound (3.13) implies the pointwise bound (4.4) for $G$. To obtain the Hölder continuity (4.5), we use that $G$ lies in $B M O_{D}(\Omega)$ and the local Hölder estimate (3.12). If we fix $x$ and $y$, then we may apply the local Hölder estimate on a local domain $\Omega_{\rho}(y)$ with radius $\rho$ comparable to $|x-y|$. The boundary estimate (4.6) follows immediately from (4.5).

Next, we turn to the symmetry property of the Green function (4.7). We let $G_{\rho}$ and $\tilde{G}_{\rho}$ be the approximate Green functions for $L$ and $L^{*}$ used in the construction of the Green function. Using the Green identity (3.15) we obtain

$$
f_{\Omega_{\rho}(y)} G_{\rho}^{\alpha \beta}(x, z) d z=f_{\Omega_{\rho}(x)} \tilde{G}_{\rho}^{\alpha \beta}(y, z) d z .
$$


Using the Hölder continuity of $G_{\rho}$ and $\tilde{G}_{\rho}$ in the second variable and the ArzelaAscoli theorem, we may extract a subsequence which converges uniformly on compact subsets of $\Omega \backslash\{x\}$. Letting $\rho$ tend to zero, we obtain $G^{\alpha \beta}(x, y)=$ $\tilde{G}^{\beta \alpha}(y, x)$.

Finally, to obtain the representation formula of the Theorem for solutions with $f$ and $f_{N}$ not zero, we may use the Arzela-Ascoli theorem to find a sequence $G_{\rho_{j}}(x, \cdot)$ which converges uniformly on $\partial \Omega$. Thus, we may take the limit in (4.9) to obtain the representation formula of the Theorem. The convergence of the integral on $\Omega$ follows since $L^{p}(\Omega) \subset H_{D}^{1}(\Omega)$ and $G_{\rho}(x, \cdot)$ converges weakly in $B M O_{D}(\Omega)$.

\section{The Green function for the Neumann prob- lem}

In this section we consider the Green function for the Neumann problem (which is the mixed problem in the extreme case where $D=\emptyset$ ). Most of our arguments parallel the construction of the Green function for the mixed problem. However, there is an additional complication. The homogeneous Neumann problem for $L$ has non-trivial solutions. Hence, we need to impose compatibility conditions on the data and conditions to guarantee uniqueness of solutions. It seems that the most natural condition for uniqueness involves the boundary values of solutions.

We let $L$ be an operator as defined in (2.3) and we consider the form $A$ now defined on $\dot{W}_{\emptyset}^{1,2}(\Omega)$, the homogeneous Sobolev space of functions with one derivative in $L^{2}(\Omega)$. Since we have chosen to norm this space by the expression $\left(\int_{\Omega}|\nabla u|^{2} d y\right)^{1 / 2}$, the elements of this space will be equivalence classes of functions under the equivalence relation $u$ is equivalent to $v$ if $u-v$ is a constant.

We let $\mathcal{V}=\left\{v: A(v, \phi)=0\right.$ for all $\left.\phi \in \dot{W}_{\emptyset}^{1,2}(\Omega)\right\}$ and $\mathcal{V}^{*}=\{v: A(\phi, v)=$ 0 for all $\left.\phi \in \dot{W}_{\emptyset}^{1,2}(\Omega)\right\}$ denote the solutions of the homogeneous Neumann problems for $L$ and $L^{*}$ respectively. Under our ellipticity assumption (2.6) and boundedness of the coefficients (2.4) we have that these spaces are finite dimensional and the Fredholm alternative implies that $\operatorname{dim} \mathcal{V}=\operatorname{dim} \mathcal{V}^{*}$. The Hölder estimate of Corollary 3.11 implies that the elements of these spaces are Hölder continuous.

We give the weak formulation of the Neumann problem for $L$

$$
\begin{cases}A(u, \phi)=-\langle f, \phi\rangle+\left\langle f_{N}, \phi\right\rangle_{\partial \Omega}, & \phi \in \dot{W}_{\emptyset}^{1,2}(\Omega) \\ u \in \dot{W}_{\emptyset}^{1,2}(\Omega) & v \in \mathcal{V} .\end{cases}
$$

If $v$ lies in $\mathcal{V}^{*}$, then we have $A(u, v)=0$ for $u \in \dot{W}_{\emptyset}^{1,2}(\Omega)$. Thus, if we are to find a solution to (5.1), we must have that $f$ and $f_{N}$ satisfy the compatibility condition

$$
\left\langle f_{N}, v\right\rangle_{\partial \Omega}-\langle f, v\rangle=0, \quad v \in \mathcal{V}^{*}
$$


For the operators we consider, the constant functions lie in $\mathcal{V}^{*}$. Thus, the first line of (5.1) is satisfied if $\phi$ is any representative of an equivalence class in $\dot{W}^{1,2}(\Omega)$.

We will use the following technical result when we construct the Green function.

Proposition 5.3 Suppose $\Omega$ is a Lipschitz domain and that the operator $L$ satisfies (2.4), (2.6), and the coerciveness condition (2.7) with $D=\partial \Omega$.

a) The norms $\|\cdot\|_{L^{2}(\Omega)}+\|\cdot\|_{\dot{W}_{\emptyset}^{1,2}(\Omega)}$ and $\|\cdot\|_{L^{2}(\partial \Omega)}$ are equivalent on the space $\mathcal{V}$.

b) If $\mu=\left(\mu^{1}, \ldots, \mu^{m}\right)$ is a finite $\mathbf{R}^{m}$-valued Borel measure on $\bar{\Omega}$, then we may find $\lambda_{\mu} \in \mathcal{V}$ so that

$$
\int_{\bar{\Omega}} v^{\alpha} d \mu^{\alpha}=\int_{\partial \Omega} \lambda_{\mu}^{\alpha} v^{\alpha} d \sigma, \quad v \in \mathcal{V}
$$

We have the estimate $\left\|\lambda_{\mu}\right\|_{L^{2}(\partial \Omega)} \leq C\|\mu\|$ where $\|\mu\|$ denotes the total variation of $\mu$.

Proof. Since $\mathcal{V} \subset \dot{W}_{\emptyset}^{1,2}(\Omega)$, it follows that $\int_{\partial \Omega}|v|^{2} d \sigma$ is finite on $\mathcal{V}$. If $v \in \mathcal{V}$ and $\int_{\partial \Omega}|v|^{2} d \sigma=0$, then $v=0$ on $\partial \Omega$ and it follows that $v$ is in the Sobolev space $\dot{W}_{\partial \Omega}^{1,2}(\Omega)$. Since we assume that the form $A$ is coercive on this space, it follows that $v=0$. Thus, we have $\|\cdot\|_{L^{2}(\partial \Omega)}$ is a norm on this space. Since $\mathcal{V}$ is finite dimensional, it follows that the norms $\|\cdot\|_{L^{2}(\Omega)}+\|\cdot\|_{\dot{W}_{\emptyset}^{1,2}(\Omega)}$ and $\|\cdot\|_{L^{2}(\partial \Omega)}$ are equivalent.

To establish part b), observe that the local boundedness estimate (3.13) implies that $v \rightarrow \int_{\bar{\Omega}} v^{\alpha} d \mu^{\alpha}$ is a continuous linear functional on $\mathcal{V}$. According to part a), $\mathcal{V}$ is a Hilbert space under the inner product $\int_{\partial \Omega} u^{\alpha} v^{\alpha} d \sigma$. Hence, the Riesz representation implies that we have a unique $\lambda_{\mu} \in \mathcal{V}$ so that

$$
\int_{\bar{\Omega}} v^{\alpha} d \mu^{\alpha}=\int_{\partial \Omega} \lambda_{\mu}^{\alpha} v^{\alpha} d \sigma
$$

Corollary 3.11 implies that the elements of $\mathcal{V}$ are bounded functions, the estimate for $\lambda_{\mu}$ follows from the Riesz representation theorem.

Next, we give a standard existence theorem for the weak Neumann problem (5.1).

Theorem 5.4 Let $\Omega$ be a Lipschitz domain and suppose that $L$ satisfies the ellipticity condition (2.6), has bounded coefficients (2.4), and that (2.7) holds for $D=\partial \Omega$. If $f$ and $f_{N}$ satisfy the compatibility condition (5.2), then the weak Neumann problem (5.1) has a unique solution and the solution u satisfies the estimate

$$
\|u\|_{L^{2}(\Omega)}+\|u\|_{\dot{W}_{\emptyset}^{1,2}(\Omega)} \leq C\left(\|f\|_{\dot{W}_{\emptyset}^{-1,2}(\Omega)}+\left\|f_{N}\right\|_{\dot{W}_{\emptyset}^{-1 / 2,2}(\partial \Omega)}\right) .
$$


Proof. The existence of solutions is a standard consequence of the Fredholm alternative. See Gilbarg and Trudinger [12, Theorem 8.6] for the proof of a similar result. To establish uniqueness, if $u$ solves (5.1) with $f=0$ and $f_{N}=0$, then we have that $u \in \mathcal{V}$. Part a) of Lemma 5.3 and the condition $\int_{\partial \Omega} u^{\alpha} v^{\alpha} d \sigma=0$ for $v \in \mathcal{V}$ imply that $u=0$.

We now give estimates for solutions of the Neumann problem to take the place of Corollary 3.16. Since the homogeneous Neumann problem may have non-constant solutions, Corollary 3.16 cannot hold for all solutions of the Neumann problem.

We consider a function $f$ in $L^{r}(\Omega), r>1$, and use Lemma 5.3 to find a function $\lambda_{f} \in \mathcal{V}^{*}$ so that the pair $f$ and $\left.\lambda_{f}\right|_{\partial \Omega}$ satisfy the compatibility condition needed to solve the Neumann problem,

$$
\left\langle\lambda_{f}, v\right\rangle_{\partial \Omega}-\langle f, v\rangle=0, \quad v \in \mathcal{V}^{*} .
$$

We let $\Lambda_{f}$ be defined by $\Lambda_{f}(\phi)=\int_{\partial \Omega} \lambda_{f}^{\alpha} \phi^{\alpha} d \sigma-\int_{\Omega} f^{\alpha} \phi^{\alpha} d y$. We observe that the constant functions lie in $\mathcal{V}^{*}$ and hence we have $\Lambda_{f}(c)=0$ for all constant vectors $c \in \mathbf{R}^{m}$. The elements in $\mathcal{V}^{*}$ are bounded and for all $p, 1 \leq p<\infty$, we have the inequalities

$$
r_{0}^{-2 / p}\|u-\bar{u}\|_{L^{p}(\Omega)}+r_{0}^{-1 / p}\|u-\bar{u}\|_{L^{p}(\partial \Omega)} \leq C\|\nabla u\|_{L^{2}(\Omega)},
$$

where $\bar{u}=f_{\Omega} u d y$. It follows that $\Lambda_{f}$ lies in $\dot{W}^{-1,2}(\Omega)$ and thus the solution of the Neumann problem

$$
\begin{cases}A(u, \phi)=\Lambda_{f}(\phi), & \phi \in \dot{W}^{1,2}(\Omega) \\ u \in \dot{W}_{\emptyset}^{1,2}(\Omega) & \\ \int_{\partial \Omega} u^{\alpha} v^{\alpha} d \sigma=0, & v \in \mathcal{V} .\end{cases}
$$

exists and satisfies $\|\nabla u\|_{L^{2}(\Omega)} \leq C\left(r, r_{0}\right)\|f\|_{L^{r}(\Omega)}$. Next, we consider the adjoint problem for $g \in L^{r}(\Omega)$,

$$
\begin{cases}A(\phi, w)=\Lambda_{g}^{*}(\phi), & \phi \in \dot{W}^{1,2}(\Omega) \\ w \in \dot{W}_{\emptyset}^{1,2}(\Omega) & \\ \int_{\partial \Omega} w^{\alpha} v^{\alpha} d \sigma=0, & v \in \mathcal{V}^{*}\end{cases}
$$

where $\Lambda_{g}^{*}(\phi)=\left\langle\lambda_{g}, \phi\right\rangle_{\partial \Omega}-\langle g, \phi\rangle$ and $\lambda_{g} \in \mathcal{V}$ satisfies $\int_{\Omega} v^{\alpha} g^{\alpha} d y=\int_{\partial \Omega} v^{\alpha} \lambda_{g}^{\alpha} d \sigma$, $v \in \mathcal{V}$. Similar considerations give the existence of a solution to the adjoint problem with $\|\nabla w\|_{L^{2}(\Omega)} \leq C\|g\|_{L^{r}(\Omega)}$. Furthermore, from (3.15) we obtain

$$
\int_{\Omega} u^{\alpha} g^{\alpha} d y=\int_{\Omega} w^{\alpha} f^{\alpha} d y
$$

The boundary integral $\int_{\partial \Omega} \lambda_{f}^{\alpha} w^{\alpha} d \sigma$ vanishes since the Neumann data $\lambda_{f}$ lies in $\mathcal{V}^{*}$ and $w$ is perpendicular to this space in $L^{2}(\partial \Omega)$. The other boundary integral vanishes for similar reasons. 
Corollary 5.10 Let $t \in\left(2, t_{0}\right)$ with $t_{0}$ as in Theorem 3.4 and $r$ be defined by $1 / r=1 / 2+1 / t$. If $f \in L^{r}(\Omega)$, then the solution of the weak Neumann problem (5.7) satisfies the estimates

$$
\|\nabla u\|_{L^{t}(\Omega)} \leq C\|f\|_{L^{r}(\Omega)}
$$

and

$$
\|u\|_{L^{r^{\prime}(\Omega)}} \leq C\|f\|_{\dot{W}^{-1, t^{\prime}}(\Omega)} .
$$

Proof. With the work above the proof is the same as Corollary 3.16. The proof of Corollary 3.16 fails at the first line, because we do not have a Poincaré inequality. Since $\Lambda_{f}(u)=\Lambda_{f}(u-\bar{u})$, we may use the estimates (5.6) to show that $\Lambda_{f}$ lies in the dual of $\dot{W}_{\emptyset}^{1,2}(\Omega)$. With $\Lambda_{f} \in \dot{W}_{\emptyset}^{1,2}(\Omega)$, the proof of this Corollary is identical to the proof of Corollary 3.16 .

Remark. For the second estimate of Corollary 5.10 to be useful, we must have that $f$ lies in $\dot{W}^{-1, t^{\prime}}(\Omega)$. In particular, we must have that the mean-value of $f$ is zero.

Corollary 5.13 If $a$ is an atom for the Hardy space $H_{\emptyset}^{1}(\Omega)$, then the solution of (5.7) with $f=a$ satisfies

$$
\|u\|_{L^{\infty}(\Omega)} \leq C
$$

Proof. Given the estimates Corollary 5.10, the result follows as in Corollary 3.20 .

We are ready to define a Green function for the the Neumann problem. We say that $G(x, \cdot)$ is a Green function for the Neumann problem with pole at $x$ if $G(x, \cdot)$ is in $L^{1}(\Omega)$ and whenever $a$ is an atom and $u$ the corresponding solution to (5.7) with $f=a$, then we have

$$
u^{\alpha}(x)=\int_{\Omega} G^{\alpha \beta}(x, y) a^{\beta}(y) d y .
$$

It is clear that the Green function is unique up to a constant.

Theorem 5.14 Let $\Omega$ be a Lipschitz domain and suppose that $L$ satisfies the ellipticity condition (2.6), has bounded coefficients (2.4), and the form for $L$ is coercive on $\dot{W}_{\partial \Omega}^{1,2}(\Omega)$. Then there exists a unique Green function $G(x, y)$ which satisfies the following estimates

$$
\begin{aligned}
& G(x, \cdot) \in B M O_{\emptyset}(\Omega) \\
& \nabla_{y} G(x, \cdot) \in L^{2}\left(\Omega \backslash \Omega_{r}(x)\right), \quad \text { for } x \in \Omega, r>0 \\
& |G(x, y)| \leq C(1+\log (d /|x-y|)), \quad x, y \in \Omega \\
& |G(x, y)-G(x, z)| \leq C \frac{|y-z|^{\gamma}}{|x-y|^{\gamma}}, \quad x, y, z \in \bar{\Omega}, \quad|y-z|<\frac{1}{2}|x-y|
\end{aligned}
$$


If $G$ and $\tilde{G}$ are the Green functions for $L$ and $L^{*}$, respectively, we may find representatives of $G$ and $\tilde{G}$ so that

$$
G^{\alpha \beta}(x, y)=\tilde{G}^{\beta \alpha}(y, x) .
$$

Furthermore, if $u$ is a solution of the weak Neumann problem with $f \in L^{p}(\Omega)$ and $f_{N} \in L^{p}(\partial \Omega)$ for some $p>1$ and satisfying the compatibility condtion (5.2), then we have

$$
u^{\alpha}(x)=\int_{\Omega} G^{\alpha \beta}(x, y) f^{\beta}(y) d y-\int_{\partial \Omega} G^{\alpha \beta}(x, y) f_{N}^{\beta}(y) d \sigma, \quad \alpha=1, \ldots, m .
$$

Proof. We fix $x \in \Omega, \alpha \in\{1, \ldots, m\}, \rho$ with $0<\rho<r_{0}$, and let $g=$ $e_{\alpha} \chi_{\Omega_{\rho}(x)} /\left|\Omega_{\rho}(x)\right|$ where $e_{\alpha}$ is the unit vector in the direction of the $\alpha$ th coordinate axis. We let $\lambda_{\rho}^{\alpha \cdot} \in \mathcal{V}$ be chosen so that $g$ and $\left.\lambda_{\rho}^{\alpha \cdot}\right|_{\partial \Omega}$ satisfy the compatibility condition needed to solve the adjoint problem (5.8). We let $G_{\rho}^{\alpha \cdot}(x, \cdot)$ be the solution of (5.8) with $g$ as above and $g_{N}$ replaced by $\lambda_{\rho}^{\alpha}$. We let $u$ be a solution of the Neumann problem (5.1) with data $f$ and $f_{N}$ in $L^{p}(\Omega)$ and $L^{p}(\partial \Omega), p>1$, respectively. From (3.15), we have

$$
f_{\Omega_{\rho}(x)} u^{\alpha}(y) d y=\int_{\partial \Omega} G_{\rho}^{\alpha \beta}(x, y) f_{N}^{\beta}(y) d y-\int_{\Omega} G_{\rho}^{\alpha \beta}(x, y) a^{\beta}(y) d y
$$

If we let $f$ be an atom and $f_{N}=0$, then Corollary 5.13 implies the left-hand side of (5.21) is bounded by a constant that is independent of $a$. It follows that $\left\|G_{\rho}^{\alpha \cdot}(x, \cdot)\right\|_{*, \emptyset} \leq C$ with $C$ independent of $\rho$. We may use compactness in the finite dimensional space $\mathcal{V}$ and the Banach-Alaoglu theorem to find $\lambda^{\alpha \cdot} \in \mathcal{V}$, $G^{\alpha \cdot}(x, \cdot) \in B M O_{\emptyset}(\Omega)$, and a sequence $\left\{\rho_{j}\right\}_{j=1}^{\infty}$ with $\lim _{j \rightarrow \infty} \rho_{j}=0$ so that $G_{\rho_{j}}^{\alpha \cdot}(x, \cdot)$ converges weakly to $G^{\alpha \cdot}(x, \cdot)$ in $B M O_{\emptyset}(\Omega)$ and $\lambda_{\rho_{j}}^{\alpha \cdot}$ converges to $\lambda^{\alpha \cdot}$ in $L^{2}(\partial \Omega)$. Combining this weak convergence of $G_{\rho_{j}}$ in $B M O_{\emptyset}(\Omega)$ with the Hölder continuity of $u$, we obtain that

$$
u^{\alpha}(x)=\int_{\Omega} G^{\alpha \beta}(x, y) a^{\beta}(y) d y .
$$

Thus, we have found our Green function and we have (5.15). From the uniqueness for the solution $u$, it follows that the limit $G(x, \cdot)$ is unique and thus we have that $G_{\rho}$ converges for all $\rho$ and not just a subsequence.

Using the Caccioppoli inequality (3.10) we can show that

$$
\int_{\Omega \backslash \Omega_{r}(x)}\left|\nabla G_{\rho}(x, y)\right|^{2} d y \leq C(r), \quad \rho<r / 2 .
$$

As this estimate is uniform in $\rho$ for $\rho$ sufficiently small, we obtain that for all $\phi \in \dot{W}_{\emptyset}^{1,2}(\Omega)$ which vanish in neighborhood of $x$ that

$$
\int_{\Omega} a_{\gamma \beta}^{i j} \frac{\partial \phi^{\beta}}{\partial y_{j}} \frac{\partial G^{\alpha \gamma}}{\partial y_{i}}(x, y) d y=\int_{\partial \Omega} \lambda^{\alpha \gamma} \phi^{\gamma} d \sigma .
$$


The pointwise estimates (5.17) and (5.18) follow from (5.15) and the local estimates in Corollary 3.11. As $G$ has non-zero Neumann data, it is important that these results allow non-zero Neumann data. The argument is identical to that of Theorem 4.1.

We establish the symmetry property (5.19). We let $G_{\rho}$ and $\tilde{G}_{\rho}$ be approximate Green functions for $L$ and $L^{*}$ as defined above. From (5.9), we obtain that

$$
f_{\Omega_{\rho}(x)} \tilde{G}_{\rho}^{\beta \alpha}(y, z) d z=f_{\Omega_{\rho}(y)} G_{\rho}^{\alpha \beta}(x, z) d z .
$$

Now, we may let $\rho$ tend to zero and obtain (5.19).

Next, we claim that the mean value $\int_{\Omega} G_{\rho}^{\alpha \beta}(x, y) d y$ is bounded for all $\rho$. As a first step, let $u$ be the solution of (5.7) with $f=e_{\beta} \chi_{\Omega}$. According to Corollary 5.10, the solution $u$ lies in $L^{r}(\Omega)$ for some $r$. As the Neumann data $\lambda_{f}$ lies in $\mathcal{V}^{*}$ and hence is bounded, we may use the estimates of Theorem 3.4 to conclude that $\nabla u$ lies in $L^{t}(\Omega)$ for some $t>2$. Since $u$ lies in $L^{r}(\Omega)$ and $\nabla u$ lies in $L^{t}(\Omega)$, we may conclude that $u$ is bounded. We apply (3.15) and obtain

$$
f_{\Omega_{\rho}(x)} u^{\alpha} d y=\int_{\Omega} G_{\rho}^{\alpha \beta}(x, y) d y
$$

Since $u$ is bounded, the claim follows. Since $G_{\rho}^{\alpha \cdot}(x, \cdot)$ is bounded in $B M O_{\emptyset}(\Omega)$ and the mean values are bounded, it follows that a subsequence of $G_{\rho}^{\alpha \cdot}(x \cdot)$ converges weakly in $L^{p}(\Omega)$ for each $p$ finite. In addition, $G_{\rho}^{\alpha \cdot}(x, \cdot)$ is a solution of $L^{*} w=0$ in a neighborhood of the boundary and the Neumann data $\lambda_{\rho}$ lies in $\mathcal{V}$ and hence is bounded. Thus, we may extract a subsequence $\left\{G_{\rho_{j}}(x, \cdot)\right\}$ which converges uniformly on $\partial \Omega$. We may let $\rho \rightarrow 0^{+}$in the representation formula (5.21) and use the continuity of $u$ to obtain the representation formula (5.20).

\section{The Green function in the plane}

In this section, we define a Green function in the plane. We will work in the homogeneous Sobolev space $\dot{W}^{1,2}\left(\mathbf{R}^{2}\right)$ which consists of functions $\phi$ with $\nabla \phi \in L^{2}\left(\mathbf{R}^{2}\right)$. We norm this space with

$$
\|\phi\|_{\dot{W}^{1,2}\left(\mathbf{R}^{2}\right)}=\left(\int_{\mathbf{R}^{2}}|\nabla \phi|^{2} d y\right)^{1 / 2} .
$$

and the elements of this Hilbert space will be equivalence classes of functions under the relation $u$ is equivalent to $v$ if $u-v$ is constant. In $\mathbf{R}^{2}$, the local domains $\Omega_{\rho}(x)$ are disks and we will use the more standard $B_{\rho}(x)=\{y$ : $|x-y|<\rho\}$ to denote these disks.

We assume that the form $A$ is coercive in the sense that

$$
A(u, u) \geq M^{-1} \int_{\mathbf{R}^{2}}|\nabla u|^{2} d y, \quad u \in \dot{W}^{1,2}\left(\mathbf{R}^{2}\right) .
$$


We may see that this condition holds for the Lamé system using an approximation argument and integration by parts.

It is an immediate consequence of the Lax-Milgram theorem that the weak formulation of the problem in the plane

$$
\left\{\begin{array}{l}
A(u, \phi)=-\langle f, \phi\rangle, \quad \phi \in \dot{W}^{1,2}\left(\mathbf{R}^{2}\right) \\
u \in \dot{W}^{1,2}\left(\mathbf{R}^{2}\right)
\end{array}\right.
$$

has a unique solution when $f$ is in the dual of $\dot{W}^{1,2}\left(\mathbf{R}^{2}\right), \dot{W}^{-1,2}\left(\mathbf{R}^{2}\right)$.

We will approximate $u$ by considering the Dirichlet problem in disks $B_{R}=$ $\{x:|x|<R\}$ for $R>0$. We let $a$ be an atom for $\mathbf{R}^{2}$ that is supported in $B_{\rho}(x)$ and for $R$ large, we let $u_{R}$ be the solution of the Dirichlet problem

$$
\left\{\begin{array}{l}
A\left(u_{R}, \phi\right)=-\int_{\Omega} a^{\alpha} \phi^{\alpha} d y \quad \phi \in \dot{W}_{\partial B_{R}}^{1,2}\left(B_{R}\right) \\
u_{R} \in \dot{W}_{\partial B_{R}}^{1,2}\left(B_{R}\right)
\end{array}\right.
$$

From Proposition 3.3 and Corollary 3.20 , we have that $\left\|u_{R}\right\|_{L^{\infty}\left(B_{R}\right)}+\left\|\nabla u_{R}\right\|_{L^{2}\left(B_{R}\right)} \leq$ $C$ and from Corollary 3.16 and Morrey's inequality (2.13)

$$
\left\|u_{R}\right\|_{L^{r^{\prime}\left(B_{R}\right)}}+\sup _{x \neq y} \frac{\left|u_{R}(x)-u_{R}(y)\right|}{|x-y|^{\gamma}} \leq C(\rho) .
$$

The Hölder index $\gamma=1-2 / t$ with $t$ and $r^{\prime}$ as in Corollary 3.16. The estimates of Corollary 3.16 are scale invariant and thus hold uniformly in $R$. The dependence on $\rho$ arises because the norm of an atom in $L^{r}\left(B_{R}\right)$ and $\dot{W}^{-1, t^{\prime}}\left(B_{R}\right)$ will depend on $\rho$. Thus, we have a function $u \in \dot{W}^{1,2}\left(\mathbf{R}^{2}\right)$ so that $\lim _{R \rightarrow \infty} u_{R}=u$ weakly in $\dot{W}^{1,2}\left(B_{S}\right)$ for each $S>0$ and $u$ solves (6.2) with $f=a$. Note that since the limiting function $u$ is unique, we have convergence for the entire family, not just a subsequence. Furthermore from the Rellich compactness theorem, we have that $u_{R}$ converges in $L^{r^{\prime}}\left(B_{S}\right)$ for each $S>0$. The Hölder estimate in (6.3) and the Arzela-Ascoli theorem imply that we also have that $u_{R}$ converges locally uniformly to $u$. According to Corollary 3.20 , the functions $u_{R}$ are uniformly bounded, hence the same holds for $u$. Thus, if $f=a$, an atom, the solution of (6.2) may be chosen so that

$$
\begin{aligned}
\|u\|_{\dot{W}^{1,2}\left(\mathbf{R}^{2}\right)}+\|u\|_{L^{\infty}\left(\mathbf{R}^{2}\right)} & \leq C \\
\|u\|_{L^{r^{\prime}}\left(\mathbf{R}^{2}\right)}+\sup _{x \neq y} \frac{|u(x)-u(y)|}{|x-y|^{\gamma}} & \leq C(\rho) .
\end{aligned}
$$

We give a definition of the Green function in the plane. We say that $G(x, \cdot)$ is a Green function in the plane for (6.2) with pole at $x$ if $G(x, \cdot)$ is in $L_{l o c}^{1}\left(\mathbf{R}^{2}\right)$ and for each atom $a$, the solution of (6.2) is given by

$$
u^{\alpha}(x)=\int_{\mathbf{R}^{2}} G^{\alpha \beta}(x, y) a^{\beta}(y) d y, \quad \alpha=1, \ldots, m .
$$

Since solutions of this weak problem are unique, it is immediate that for each $x, G(x, \cdot)$ is a unique element of $B M O\left(\mathbf{R}^{2}\right)$. In other words, $G(x, \cdot)$ is unique up to a constant. 
Finally, we give a theorem which establishes existence and regularity of the Green function in $\mathbf{R}^{2}$.

Theorem 6.7 If L satisfies (2.4) and (6.1), then there is a unique Green function $G(x, \cdot)$ with pole at $x$ and the Green function satisfies

$$
\begin{gathered}
G(x, \cdot) \in B M O\left(\mathbf{R}^{2}\right) \\
\nabla_{y} G(x, \cdot) \in L_{l o c}^{2}\left(\mathbf{R}^{2} \backslash\{x\}\right) \\
\left|G(x, y)-f_{B_{1}(x)} G(x, z) d z\right| \leq C(1+|\log (|x-y|)|), \quad x, y \in \mathbf{R}^{2} \\
|G(x, y)-G(x, z)| \leq C \frac{|y-z|^{\gamma}}{|x-y|^{\gamma}}, \quad \text { if }|y-z|<\frac{1}{2}|x-y|
\end{gathered}
$$

The exponent $\gamma$ is as in Corollary [3.11 and $C$ may be chosen to depend on $M$ and $\gamma$.

Finally, if $G$ and $\tilde{G}$ are the Green functions in the plane for the operators $L$ and $L^{*}$, then we may find representatives which satisfy the symmetry condition

$$
G^{\alpha \beta}(x, y)=\tilde{G}^{\beta \alpha}(y, x) .
$$

Proof. To construct the Green function $G(x, \cdot)$ we fix $x, \rho>0$, and let $f_{\rho}=$ $\frac{1}{\pi \rho^{2}} \chi_{B_{\rho}(x)}-\frac{\rho^{2}}{3 \pi} \chi_{B_{2 / \rho}(x) \backslash B_{1 / \rho}(x)}$. As $f_{\rho} e_{\alpha}$ is an atom (though the constant depends on $\rho$ ), we may let $G_{\rho}(x, \cdot)$ be the weak solution of the equation $L^{*} G_{\rho}^{\alpha \cdot}(x, \cdot)=$ $f_{\rho} e_{\alpha}$. We let $a$ be an atom and $u$ the solution of $L u=a$ constructed above. As in the proof of (3.15), the weak formulations of the equations satisfied by $u$ and $G_{\rho}^{\alpha \cdot}(x, \cdot)$ give

$$
A\left(u, G_{\rho}^{\alpha \cdot}(x, \cdot)\right)=\int_{\mathbf{R}^{2}} G_{\rho}^{\alpha \beta}(x, y) a^{\beta}(y) d y=\int_{\mathbf{R}^{2}} f_{\rho} u^{\alpha} d y .
$$

The estimate for $\|u\|_{L^{\infty}\left(\mathbf{R}^{2}\right)}$ in (6.4) implies that $\left|\int G_{\rho}^{\alpha \beta}(x, \cdot) a^{\beta} d y\right|=\left|\int f_{\rho} u_{\alpha} d y\right| \leq$ $C$. Now, we may conclude from (2.10) that $G_{\rho}(x, \cdot)$ lies in $B M O\left(\mathbf{R}^{2}\right)$. Thus, we may use the Banach-Alaoglu Theorem to find a function $G(x, \cdot)$ and a sequence $\left\{G_{\rho_{k}}(x, \cdot)\right\}$ with $\lim _{k \rightarrow \infty} \rho_{k}=0$ so that $G_{\rho_{k}}(x, \cdot)$ converges to $G(x, \cdot)$ in the weak-* topology of $B M O\left(\mathbf{R}^{2}\right)$. From the estimates in (6.5), it follows that

$$
u^{\alpha}(x)=\lim _{\rho \rightarrow 0^{+}} \int_{\mathbf{R}^{2}} f_{\rho} u^{\alpha} d y .
$$

Hence, we obtain the representation formula (6.6). We may use Caccioppoli's inequality (3.10) to obtain uniform bounds on $\nabla_{y} G_{R}(x, \cdot)$ in $L^{2}$ of compact subsets of $\mathbf{R}^{2} \backslash\{x\}$. Thus $G(x, \cdot)$ is a solution of $L^{*} G(x, \cdot)=0$ in $\mathbf{R}^{2} \backslash\{x\}$. Now the pointwise estimates (6.10) and (6.11) follow as they do for the mixed problem.

Finally, we establish the symmetry property (6.12). As our construction of $G$, stands we have no information about the behavior of $G$ in the first variable. 
We begin by claiming that we can fix a representative of $G(x, \cdot)$ so that $G$ is locally integrable in $\mathbf{R}^{2} \times \mathbf{R}^{2}$. Towards this end, we observe that if we fix $x$ in $\mathbf{R}^{2}$ and let $h_{\rho, x}=\frac{1}{\pi \rho^{2}}\left(\chi_{B_{\rho}(x)}-\chi_{B_{\rho}(0)}\right)$, then $e_{\alpha} h_{\rho, x}$ lies in $\dot{W}^{-1, t^{\prime}}\left(\mathbf{R}^{2}\right)$, the dual of $\dot{W}^{1, t}\left(\mathbf{R}^{2}\right), t \geq 2$, with $\left\|h_{\rho} e_{\alpha}\right\|_{\dot{W}^{-1, t^{\prime}}\left(\mathbf{R}^{2}\right)} \leq C|x|^{1-2 / t}$. We let $v_{\rho}(x, \cdot)$ be the solution of $L^{*} v_{\rho}(x, \cdot)=e_{\alpha} h_{\rho, x}$. Using the estimates of Corollary 3.16, we may show that the map $x \rightarrow v_{\rho}(x, \cdot)$ is a continuous map from $\mathbf{R}^{2}$ into $L^{r^{\prime}}\left(\mathbf{R}^{2}\right)$ where $r^{\prime}$ is as in (3.18). If we fix a representative of $G_{\rho}(0, \cdot)$, the function $G_{\rho}(0, \cdot)+v_{\rho}(x, \cdot)$ gives an approximate Green function that is locally integrable in $\mathbf{R}^{2} \times \mathbf{R}^{2}$. If we let $\rho \rightarrow 0^{+}$, we obtain the same conclusion for $G$.

We let $G$ and $\tilde{G}$ be the Green functions as constructed in the previous paragraph for $L$ and $L^{*}$. We fix atoms $a$ and $b$ and let $u$ and $v$ solve the equations $L u=a$ and $L^{*} v=b$. From the weak formulation (6.2), we have $A(u, v)=\int u^{\alpha} b^{\alpha} d y=\int a^{\beta} v^{\beta} d y$. Using the representation (6.6) and Fubini's theorem, we obtain

$$
\int_{\mathbf{R}^{2} \times \mathbf{R}^{2}} b^{\alpha}(x) G^{\alpha \beta}(x, y) a^{\beta}(y) d x d y=\int_{\mathbf{R}^{2} \times \mathbf{R}^{2}} b^{\alpha}(x) \tilde{G}^{\beta \alpha}(y, x) a^{\beta}(y) d x d y .
$$

As this holds for all atoms $a$ and $b$, we have functions $\phi$ and $\tilde{\phi}$ so that

$$
G(x, y)+\phi(x)=\tilde{G}(y, x)+\phi(y) .
$$

\section{References}

[1] P. Auscher, A. McIntosh, and P. Tchamitchian. Heat kernels of second order complex elliptic operators and applications. J. Funct. Anal., 152(1):2273, 1998.

[2] M. Calanchi, L. Rodino, and M.N. Tri. Solutions of logarithmic type for elliptic and hypoelliptic equations. In Proceedings of the Conference "Differential Equations" (Italian) (Ferrara, 1996), volume 41, suppl., pages 111-127 (1997), 1996.

[3] D.C. Chang. The dual of Hardy spaces on a bounded domain in $\mathbf{R}^{n}$. Forum Math., 6(1):65-81, 1994.

[4] S. Chanillo and Y.Y. Li. Continuity of solutions of uniformly elliptic equations in $\mathbf{R}^{2}$. Manuscripta Math., 77(4):415-433, 1992.

[5] J. Choi and S. Kim. Neumann functions for second order elliptic systems with measurable coefficients. arXiv:1112.2436v1.

[6] R.R. Coifman and G. Weiss. Extensions of Hardy spaces and their use in analysis. Bull. Amer. Math. Soc., 83:569-645, 1976.

[7] G. Dolzmann and S. Müller. Estimates for Green's matrices of elliptic systems by $L^{p}$ theory. Manuscripta Math., 88(2):261-273, 1995. 
[8] H. Dong and S. Kim. Green's matrices of second order elliptic systems with measurable coefficients in two dimensional domains. Trans. Amer. Math. Soc., 361(6):3303-3323, 2009.

[9] F. W. Gehring. The $L^{p}$-integrability of the partial derivatives of a quasiconformal mapping. Acta Math., 130:265-277, 1973.

[10] M. Giaquinta. Multiple integrals in the calculus of variations and nonlinear elliptic systems, volume 105 of Annals of Mathematics Studies. Princeton University Press, Princeton, NJ, 1983.

[11] M. Giaquinta and G. Modica. Regularity results for some classes of higher order nonlinear elliptic systems. J. Reine Angew. Math., 311/312:145-169, 1979 .

[12] D. Gilbarg and N.S. Trudinger. Elliptic partial differential equations of second order. Springer-Verlag, Berlin, 1983.

[13] K. Gröger. A $W^{1, p}$-estimate for solutions to mixed boundary value problems for second order elliptic differential equations. Math. Ann., 283(4):679-687, 1989.

[14] M. Grüter and K.O. Widman. The Green function for uniformly elliptic equations. Manuscripta Math., 37(3):303-342, 1982.

[15] J.L. Journé. Calderón-Zygmund operators, pseudodifferential operators and the Cauchy integral of Calderón, volume 994 of Lecture Notes in Mathematics. Springer-Verlag, Berlin, 1983.

[16] C.E. Kenig and W.M. Ni. On the elliptic equation $L u-k+K \exp [2 u]=0$. Ann. Scuola Norm. Sup. Pisa Cl. Sci. (4), 12(2):191-224, 1985.

[17] C.E. Kenig and J. Pipher. The Neumann problem for elliptic equations with nonsmooth coefficients. Invent. Math., 113:447-509, 1993.

[18] W. Littman, G. Stampacchia, and H. Weinberger. Regular points for elliptic equations with discontinuous coefficients. Ann. della Sc. N. Sup. Pisa, 17:45-79, 1963.

[19] N.G. Meyers. An $L^{p}$-estimate for the gradient of solutions of second order elliptic divergence equations. Ann. Scuola Norm. Sup. Pisa (3), 17:189206, 1963.

[20] D. Mitrea and I. Mitrea. On the regularity of Green functions in Lipschitz domains. Comm. Partial Differential Equations, 36(2):304-327, 2011.

[21] C.B. Morrey, Jr. On the solutions of quasi-linear elliptic partial differential equations. Trans. Amer. Math. Soc., 43(1):126-166, 1938.

[22] O. A. Oleĭnik, A. S. Shamaev, and G. A. Yosifian. Mathematical problems in elasticity and homogenization, volume 26 of Studies in Mathematics and its Applications. North-Holland Publishing Co., Amsterdam, 1992.

[23] K.A. Ott and R.M. Brown. The mixed problem for the Laplacian in Lipschitz domains. arXiv:0909.0061 [math.AP], 2009. 
[24] G. Stampacchia. Problemi al contorno ellitici, con dati discontinui, dotati di soluzionie hölderiane. Ann. Mat. Pura Appl. (4), 51:1-37, 1960.

[25] J.L. Taylor, K.A. Ott, and R.M. Brown. The mixed problem in Lipschitz domains with general decompositions of the boundary. To appear, Trans. Amer. Math. Soc.

May 8, 2012 